\title{
Local Structural Investigation of Hafnia-Zirconia Polymorphs in Pow- ders and Thin Films by X-ray Absorption Spectroscopy
}

Tony Schenk ${ }^{1,+}{ }^{*}$, Andris Anspoks ${ }^{2,}{ }^{*}$, Inga Jonane ${ }^{2}$, Reinis Ignatans ${ }^{3}$, Brienne S. Johnson ${ }^{4}$, Jacob L. Jones ${ }^{4}$, Massimo Tallarida ${ }^{5}$, Carlo Marini ${ }^{5}$, Laura Simonelli ${ }^{5}$, Philipp Hönicke ${ }^{6}$, Claudia Richter ${ }^{1}$, Thomas Mikolajick $^{1,7}$, Uwe Schroeder ${ }^{1}$

${ }^{1}$ NaMLab gGmbH/TU Dresden, Noethnitzer Str. 64, D-01187 Dresden, Germany

2 Institute of Solid State Physics, University of Latvia, Kengaraga street 8, 1063 Riga, Latvia

${ }^{3}$ Institute of Materials, École polytechnique fédérale de Lausanne, CH-1015 Lausanne, Switzerland

${ }^{4}$ Department of Materials Science and Engineering, NC State University, Raleigh, NC 27695-7907, USA

${ }^{5}$ ALBA Synchrotron, Carrer de la llum 2-20, 08290, Cerdanyola del Vallès, Barcelona, Spain

${ }^{6}$ Physikalisch-Technische Bundesanstalt (PTB), Abbestr. 2-12, 10587 Berlin, Germany

${ }^{7}$ Institute of Semiconductors and Microsystems, TU Dresden, D-01062 Dresden, Germany

${ }^{+}$currently with Materials Research and Technology Department, Luxembourg Institute of Science and Technology (LIST), 41 Rue du Brill, L-4422 Belvaux, Luxembourg

*E-mail: tony.schenk@list.lu; aanspoks@cfi.lu.Iv

\begin{abstract}
Despite increasing attention for the recently found ferro- and antiferroelectric properties, the polymorphism in hafnia- and zirconia-based thin films is still not sufficiently understood. In the present work, we show that it is important to have a good quality X-ray absorption spectrum to go beyond an analysis of the only the first coordination shell. Equally important is to analyze both EXAFS and XANES spectra in combination with theoretical modelling to distinguish the relevant phases even in bulk materials and to separate structural from chemical effects. As a first step toward the analysis of thin films, we start with the analysis of bulk references. After that, we successfully demonstrate an approach that allows us to extract high-quality spectra also for $20 \mathrm{~nm}$ thin films. Our analysis extends to the second coordination shell and includes effects created by chemical substitution of $\mathrm{Hf}$ with Zr to unambiguously discriminate the different polymorphs. The trends derived from X-ray absorption spectroscopy agree well with $\mathrm{X}$-ray diffraction measurements. In this work we clearly identify a gradual transformation from monoclinic to tetragonal phase as the $\mathrm{Zr}$ content of the films increases. We separated structural effects from effects created by chemical disorder when ration of $\mathrm{Hf}: \mathrm{Zr}$ is varied and found differences for the incorporation of the substitute atoms between powders and thin films, which we attribute to the different fabrication routes. This work opens the door for further in-depth structural studies to shine light into the chemistry and physics of these novel ferroelectric thin films that show high application relevance.
\end{abstract}

KEYWORDS: extended X-ray absorption fine structure; X-ray absorption near edge structure; ferroelectrics; hafnium oxide; zirconium oxide 


\section{INTRODUCTION}

The polymorphism of hafnia and zirconia has been intensely studied, first as ceramics in the frame of refractories ${ }^{1,2}$ and solid electrolytes (oxygen conduction) ${ }^{3,4}$, transformation toughening ("ceramic steel") ) $^{5,6}$ and later, in the quest for so-called "high-k dielectrics" (dielectrics with higher relative permittivity than 3.9) to replace $\mathrm{SiO}_{2}$ in both transistors and capacitors of semiconductor industry ${ }^{8,9}$. This is why a 2011 publication by Böscke et al. ${ }^{10}$ claiming ferroelectric behavior in $10 \mathrm{~nm}$ thick $\mathrm{Si}: \mathrm{HfO}_{2}$ films took researchers by surprise. The understandable doubts steadily reduced in the following years as reports of similar properties induced by a wealth of dopants and studies by different groups using unique fabrication methods and various electrode materials suggested some universality at least for thin film samples. ${ }^{11,12,13,14,15,16,17,18,19,20}$ Further "must-haves" for a ferroelectric, beyond a hysteretic curve of polarization $P$ vs. electric field $E$, such as counter-clockwise switching in a ferroelectric field-effect transistor (FE-FET) $)^{21,22}$, piezo- ${ }^{10,23}$ and pyroelectricity ${ }^{24,25}$, as well as the experimental proof of the presence of a polar phase ${ }^{26,27}$, solidified the original claim of Böscke et al. ${ }^{10}$. The implementation of these new class of fluorite-type ferroelectrics in first devices for memory (transistor ${ }^{21}$ and capacitor ${ }^{28}$ based), energy harvesting and electrocaloric cooling $^{29}$ or supercapacitors ${ }^{30,31}$ applications was even faster than the actual proof of the claimed phase behind. In recent years the pool of applications has been expanded by the new concept of antiferroelectric memory ${ }^{32}$, neuromorphic devices ${ }^{33,34}$ negative capacitance FETs ${ }^{35}$, ferroelectric tunnel junctions ${ }^{36}$ as well as tunable microwave filters and phase shifters ${ }^{37,38}$.

Nonetheless, fundamental material research could not keep pace with this encouraging development. This is due to the following aspects:

1) Most of the utilized films are polycrystalline and contain a mixture of phases. A wealth of potential polymorphs exists and especially the many orthorhombic phases are only distinguished by the oxygen sublattice ${ }^{26}$, which is not easily accessible via standard X-ray diffraction (XRD) methods.

2) A size effect is involved in the occurrence of ferroelectricity ${ }^{39,40,41}$. Most of the ferroelectric films are less than $30 \mathrm{~nm}$ thick and even for epitaxial films, the orthorhombic phase relaxes into the monoclinic bulk phase beyond a certain thickness. ${ }^{18,42,43,44,45}$

3) Also from theory, i.e. ab-initio simulations, bulk calculations cannot explain the observed ferroelectricity. Computation-intense "real world" parameters like dopants, vacancies, strain or surface energy have been included to come closer to an explanation, but the work is still ongoing. ${ }^{39,46,47,48}$

Up to now, the best insights into the structure are provided by high-resolution, aberration-corrected transmission electron microscopy (TEM) methods. ${ }^{26,27,49,50}$ However, thin lamellas have to be cut for TEM analysis, which raises the questions if the film still remains unchanged. Moreover, sampling is poor in polycrystalline films as only grains that, by chance, are oriented in a lower-index axis toward the beam can be analyzed. Here, extended X-ray absorption fine structure (EXAFS) analysis comes into play. This method is suited to study the application relevant capacitor stacks of a dielectric film sandwiched between two electrodes as it is used in ferroelectric memories.

X-ray absorption spectroscopy (XAS) is an atomic site-specific probe ${ }^{51}$, which, differently from XRD, does not require any long-range crystal symmetry and hence can be used to directly access the local atomic structure of matter, including crystalline and amorphous states. By EXAFS it is possible to quantify interatomic distances, the kind and the number of surrounding atoms as well as their disorder around an absorber, and it is particularly suited to investigate local strain. ${ }^{52,53,54,51}$ In future, EXAFS could be a very powerful method to study all applicationrelevant pre-conditioning or degradation ${ }^{55,49,56}$ mechanisms.

However, strong parameter correlations limit the investigation of complex problems and the data analysis relies on the best possible choice of boundary conditions and initial guesses. Here, a look into literature is the first step and there is already a solid body of EXAFS data for hafnia and zirconia available $57,58,59,60,61,62,63$. Very comprehensive studies of zirconia polymorphs and effect of various dopants was performed by Li et al. ${ }^{57,64,65,66,67,68}$. In these studies, a quantitative analysis of EXAFS data are performed including the first coordination shell (metal-oxygen, Me$\mathrm{O}$ ) and second coordination shell (metal-metal, Me-Me). What all studies have in common is that the Me-O and Me-Me distances were grouped into some representative mean value(s). This is mainly done because of the nontrivial structure of the material with many degrees of freedom for the low symmetry lattices, which leads to too 
many correlated free parameters (coordination numbers, relative distances, mean-square relative displacements often called Debye-Waller factors).

This work starts with a section on theoretical modelling of XAS spectra for the concerned polymorphs and the impact of chemical substitution of $\mathrm{Hf}$ with $\mathrm{Zr}$. In the experimental part, we first analyze powder samples of different phases to establish an understanding of the bulk reference phases and their characteristic features. After that, thin films of different dielectric behavior and corresponding structure were approached. Here, the thin film nature, again, warranted some deeper thought regarding sample preparation. In preliminary experiments, the simplest approach of using fluorescence mode to measure $10 \mathrm{~nm}$ thin films on $12 \mathrm{~nm}$ TiN and Si wafer piece resulted in very low signal quality insufficient for the aforementioned analysis of further coordination shells. Fluorescence mode also suffers the issue of self-absorption, which can in principle be corrected for, but not always sufficiently. ${ }^{69}$ It was thus, decided to use 1) transmission mode, 2) $20 \mathrm{~nm}$ thick dielectric films and 3) a thinner and lighter substrate of $125 \mu \mathrm{m}$ rigid graphite that allows to 4) pile multiple of these capacitor stacks on top of one another. Moreover, instead of hafnia doped by Si or other elements, the binary solid solution of hafnia and zirconia was chosen, to increase the likelihood of obtaining high-quality spectra of two metal edges to be combined in the subsequent analysis. With this approach we were able to achieve a comparably high signal quality for both ceramics and thin film samples.

\section{MODELLING OF XAS}

To understand the complexity of EXAFS analysis we have calculated the theoretical configuration-averaged EXAFS spectra for the monoclinic $\left(\mathrm{P} 2_{1} / \mathrm{c}\right)$, orthorhombic $\left(\mathrm{Pca}_{2}\right)$ and tetragonal $\left(\mathrm{P}_{2} / \mathrm{nmc}\right) \mathrm{HfO}_{2}$ and $\mathrm{ZrO}_{2}$ phases. Unit cells as provided by Materlik et al. ${ }^{39}$, were used in the software FEFF $9^{70}$. The spectra were averaged over all atomic positions, which is especially important when modelling the substitution of $\mathrm{Hf}$ with $\mathrm{Zr}$. Fourier transform of EXAFS (FT-EXAFS) here and afterwards is performed using a Kaiser-Bessel window in the $k$-range $3-13 \AA^{-1}$ to be comparable with experimental data. In the calculations reported in Figure 1, thermal and static disorder effects are neglected, which results in much larger amplitudes at high wavenumber values $k$ than in a real spectrum. Nonetheless, the $k$-position of the oscillations are unaffected and reliably reflect what trends to expect in a real spectrum. At low wavenumber values (up to $6 \AA^{-1}$ ), the signal from the first coordination shell (generally Me-O, here $\mathrm{Hf}-\mathrm{O}$ and $\mathrm{Zr}-\mathrm{O}$ ) dominates. It is rather close for all polymorphs as it originates from a rather broad distribution of distances. This is especially true for low symmetry phases (e.g. monoclinic or orthorhombic) where it is very hard to distinguish strained phases in thin films just by Me-O distances. ${ }^{61}$ Spectral weight observed beyond $10 \AA^{-1}$ stems mainly from the $\mathrm{Hf}-\mathrm{Hf}$ and $\mathrm{Zr}-\mathrm{Zr}$ pairs. In this shell, the differences between the three phases are much more pronounced, as the metal positions define the Bravais lattice system - monoclinic, orthorhombic or tetragonal. The tetragonal phase for example, has the most pronounced Me-Me features in the FT-EXAFS plots (Fig. 1c). 
EXAFS Simulations for $\mathrm{HfO}_{2}$ and $\mathrm{ZrO}_{2}$ Polymorphs
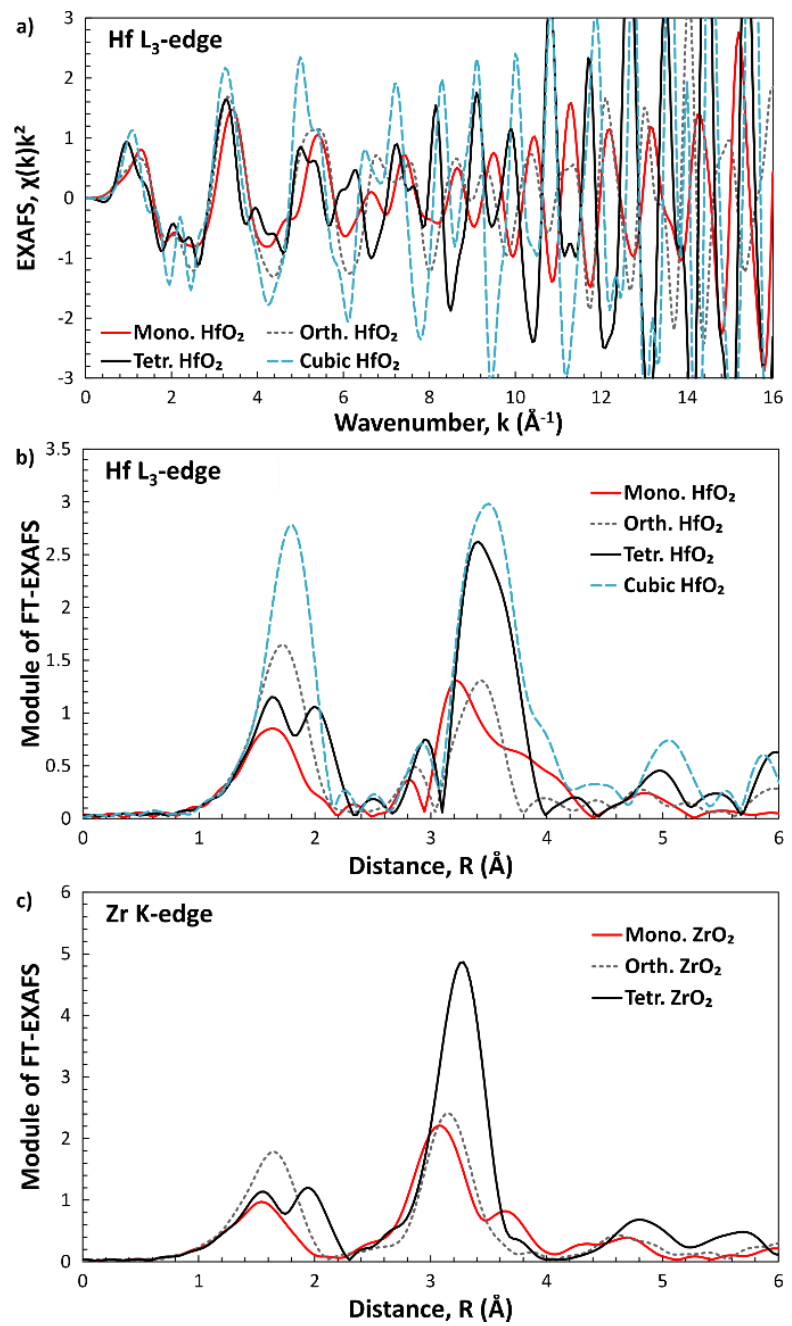

Figure 1. Simulated configuration-averaged EXAFS for $\mathrm{Hf}_{3}$-edge of $\mathrm{HfO}_{2}$ polymorphs (monoclinic $\mathrm{P} 2_{1} / \mathrm{c}$, orthorhombic Pca2 ${ }_{1}$, tetragonal $\mathrm{P} 4_{2} / \mathrm{nmc}$ and cubic $\mathrm{Fm} \overline{3} \mathrm{~m}$ ) from Materlik et al. ${ }^{39}$ (a) their Fourier transform of EXAFS (FT-EXAFS) (b), and corresponding $\mathrm{ZrO}_{2}$ polymorphs with exactly the same structure (c).

Also, in the Fourier transform of the calculated EXAFS spectra (Figure 1b) the spectral differences of the three polymorphs are more pronounced in the second coordination shell, i.e. the metal-metal (Me-Me) distances. Only the tetragonal structure possesses a specific fingerprint for the first coordination shell (Hf-O) seen as double peak at 1.7 and $2 \AA$. In order to distinguish between polymorphs, we believe it is fundamental to extend the EXAFS analysis beyond the first coordination shell as strain effects might also account for shifts to higher or lower distances. ${ }^{71,44,72,73}$

Moreover, there are many nonequivalent Me-Me distances for low symmetry structures, which poses a challenge for theoretical calculations and interpretation of the experimental results. The maximum number $\mathrm{N}$ of independent parameter in an EXAFS fit is limited by the window in wavenumber $k$ and distance $R$ used $(N=\Delta k \cdot \Delta R \cdot 2 / \pi)$. Trying to fit experimental signals with a larger number of free parameters leads to fake results. For this reason, in most of the cases, the Me-O distances are approximated by one or two aggregated average distances and Me-Me distance by one to three effective distances. ${ }^{57,58,61,62}$ The distribution of the distances might also stem from strains occurring in thin films and doped materials ${ }^{50,71,44,72,73,74}$ which complicates the analysis even more making the discrimination of the different phases of $\mathrm{Hf}_{1-\mathrm{x}} \mathrm{Zr}_{\mathrm{x}} \mathrm{O}_{2}$ compounds a serious challenge. Therefore, spectra with high data quality beyond $10 \AA^{-1}$ are mandatory. This is a challenging experimental task as data at high $k$ values are damped by static and thermal disorder, and sometimes limited by experimental noise. 
An important difference seen in FT-EXAFS for $\mathrm{Zr}$ K-edge compared to $\mathrm{Hf}_{3}$-edge spectra of the pure oxides are sharper (narrower and with higher amplitude) features corresponding to the second coordination shell of the metal for the $\mathrm{Zr}$ - $\mathrm{Zr}$ compared to the $\mathrm{Hf}-\mathrm{Hf}$ for identical structures in all phases. This is a consequence of different values and shape for photoelectron effective back scattering amplitude $\left(F_{j}(k)\right)$ and phase shift $\left(\Phi_{j}(k)\right)$ as a function of wavevector $(k)$ (see Figure S17 in the Supporting Information) $)^{51}$ :

$\chi(k)=S_{O}^{2} \sum_{j} \frac{N_{j}}{k r_{j}^{2}} F_{j}(k) \exp \left(-2 \sigma_{j}^{2} k^{2}\right) \sin \left(2 k r_{j}+\Phi_{j}(k)\right)$,

where $\chi(k)$ is EXAFS signal, $r_{j}$ is distance between absorbing atom and backscattering atom $j, N_{j}$ is a number of the atoms $j$ at the distance $r_{j}, \sigma_{j}^{2}$ is a mean square relative displacement (variation of the $r_{j}$ due to static and thermal disorder, sometimes called Debye-Waller factor), $F_{j}(k)$ and $\Phi_{j}(k)$ are effective back scattering amplitude and phase shift for atom pair absorbing atom-atom $j, S_{o}^{2}$ is an amplitude reduction factor. These differences seen in the FT-EXAFS and EXAFS spectra for identical structures of $\mathrm{HfO}_{2}$ and $\mathrm{ZrO}_{2}$ should be considered when analysing experimental spectra. Without this knowledge one can easily come to misleading conclusions that the $\mathrm{Zr}-\mathrm{Zr}$ distances have smaller variations than $\mathrm{Hf}-\mathrm{Hf}$ ones.

\section{Impact of Zr Content on Simulated EXAFS Spectra}
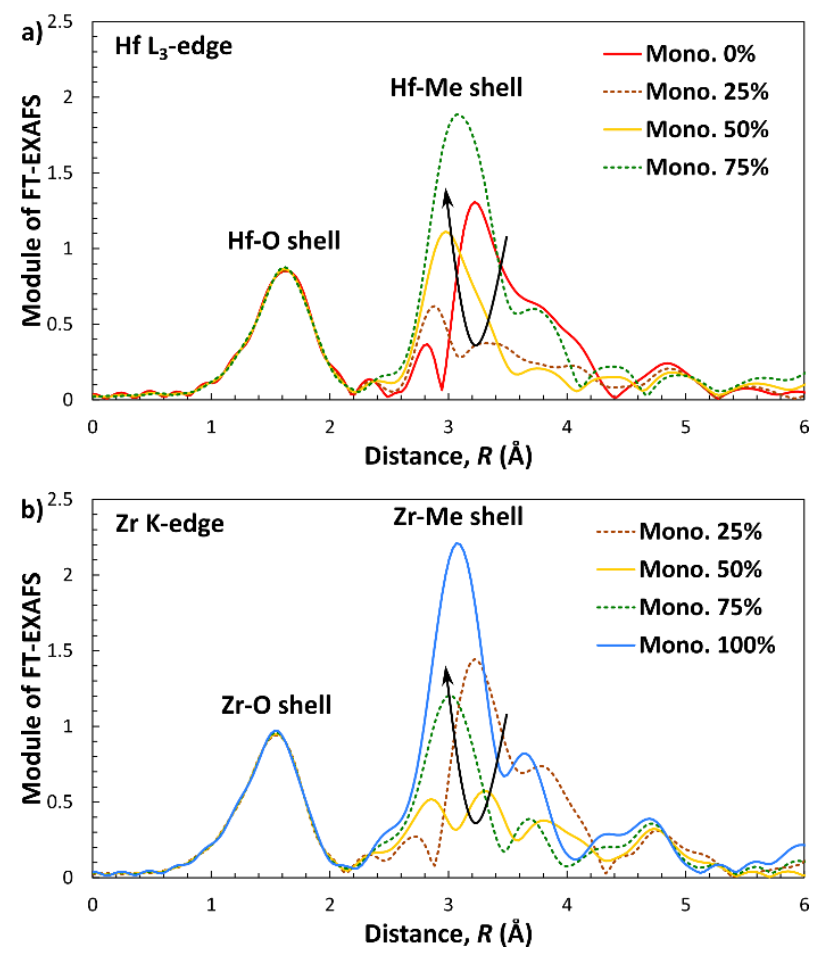

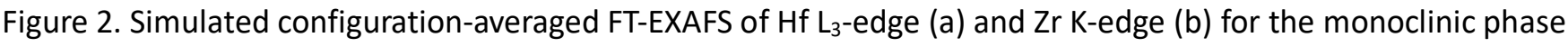
of $\mathrm{Hf}_{1-x} \mathrm{Zr}_{x} \mathrm{O}_{2}$ compounds with different $\mathrm{Zr}$ concentrations (percentages denote the $\mathrm{Zr}$ content). The arrow indicates the changes found for increasing $Z r$ content (red via orange, yellow and green to blue line): first the characteristic Me-Me features caused by interactions with surrounding $\mathrm{Hf}$ atoms (red line) get damped out, then the characteristic features from surrounding $\mathrm{Zr}$ atoms (blue line) appear. The corresponding simulation data for the orthorhombic and tetragonal phase as well as the plot of the EXAFS signal can be found in Figures S13 and S14 in the SI.

Other important outcome from this formula is that the features (peaks) in FT-EXAFS spectrum that correspond to the coordination shells (like $\mathrm{Hf}-\mathrm{O}$ or $\mathrm{Hf}-\mathrm{Hf}$ ) are distorted due the effective back scattering amplitude $\left(F_{j}(k)\right)$ and phase shift $\left(\Phi_{j}(k)\right)$ which are highly nonlinear functions (Figure S17 in the Supporting Information), so the shape and distance do not correspond to the pair distribution functions. As these phase shifts are specific to every chemical element, it also not possible to speculate about for example $\mathrm{Zr}-\mathrm{Zr}$ and $\mathrm{Hf}-\mathrm{Hf}$ distances just comparing the FT- 
EXAFS peaks. At the same time for polymorphs of the same chemical composition shift in the peak positions in FTEXAFS can be related with the real shift of the average distances, only change in disorder also should be taken into account.

In Figure 2, we modelled the substitution of the $\mathrm{Hf}$ with $\mathrm{Zr}$ for the monoclinic phase. The $\mathrm{Hf}$ atoms were replaced in a systematic way with $\mathrm{Zr}$ (all possible combinations) and made an average of the obtained signal from all nonequivalent atomic positions.

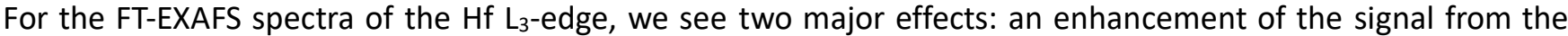
second coordination shell ( $\mathrm{Hf}-\mathrm{Me}$ ) and a shift of the $\mathrm{Hf}-\mathrm{Me}$ feature to shorter distances as a consequence of different photoelectron backscattering amplitude and phase shift of $\mathrm{Zr}$ compared to $\mathrm{Hf}$. The amplitude of $\mathrm{Hf}-\mathrm{Me}$ feature in FT-EXAFS decreases for smaller concentrations of $\mathrm{Zr}$ as consequence of chemical disorder causing interference of the photoelectron signals from $\mathrm{Hf}-\mathrm{Hf}$ and $\mathrm{Hf}$ - $\mathrm{Zr}$ interactions. The lowest amplitude of the second shell features are observed for $25 \% \mathrm{Zr}$.

Exactly the same effect is observed for the $\mathrm{Zr}$ K-edge in Figure $2 \mathrm{~b}$. However, we note that in case of an even random distribution of $\mathrm{Zr}$ and $\mathrm{Hf}$ atoms, the $\mathrm{Hf}-\mathrm{Me}$ feature in the FT-EXAFS is very diffuse in case of $50 \%$ concentration of $\mathrm{Zr}$ and $\mathrm{Hf}$, while it is sharper for the $\mathrm{Hf}_{\mathrm{L}_{3}}$-edge. The transformation between the spectra of the pure oxide toward what is seen for the largest admixture of the "foreign" metal atom, occurs earlier for the Hf than for the $\mathrm{Zr}$ edge.

\section{Simulated O K-edge XANES Spectra}
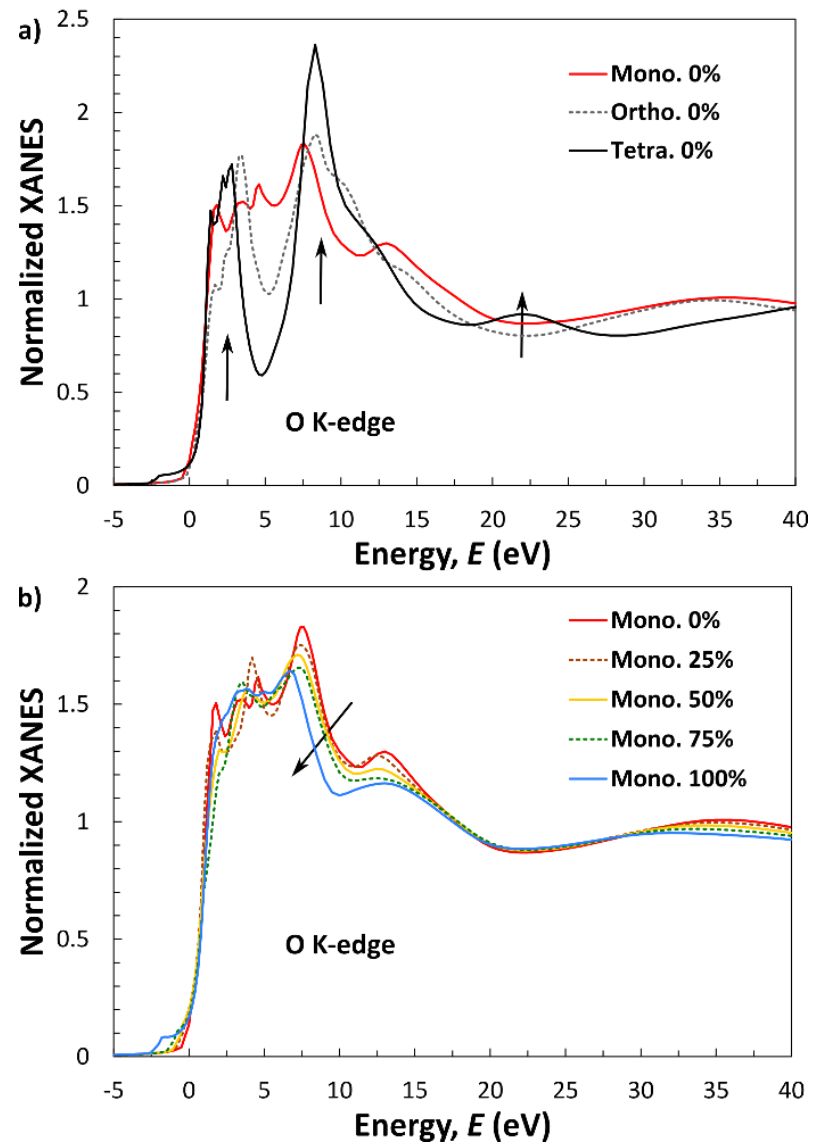

Figure 3. Calculated configuration-averaged XANES spectra of the $\mathrm{O}$ K-edge for the monoclinic $P 2_{1} / c$, orthorhombic $\mathrm{Pca}_{1}$ and tetragonal $\mathrm{P}_{2} / \mathrm{nmc}$ polymorphs of pure $\mathrm{HfO}_{2}(\mathrm{a})$, and the effect of $\mathrm{Hf}$ substitution with $\mathrm{Zr}$ for monoclinic $\mathrm{Hf}_{1-\mathrm{x}} \mathrm{Zr}_{\mathrm{x}} \mathrm{O}_{2}$. The energy scale is relative to the absorption edge.

Moreover, O K-edge XANES simulations (Figure 3) were performed using FDMNES code (version of March 2019) ${ }^{75}$ and the same configuration averaging of the spectra for all non-equivalent atomic positions and all possible $\mathrm{Zr}$ 
substitutions of Hf. We used the finite difference method, which is more suited for soft X-ray XANES calculations than Green function formalism ${ }^{75}$, and self-consistent potential calculations. We calculated XANES spectra for clusters with the radius $6.42 \AA$ (monoclinic) and $8 \AA$ (orthorhombic and tetragonal) for all non-equivalent O positions with subsequent configuration averaging of the spectra.

In the $\mathrm{O}$ K-edge XANES of Figure $3 \mathrm{a}$, we see an enhancement of two peaks corresponding to the empty $\mathrm{e}_{\mathrm{g}}$ and $\mathrm{t}_{2 \mathrm{~g}}$ states of the $\mathrm{Hf} 5 \mathrm{~d}$ or the $\mathrm{Zr} 4 \mathrm{~d}$ orbitals ${ }^{61}$ with increasing symmetry. The tetragonal phase has the most pronounced features, but also a difference between the monoclinic and the orthorhombic phase is seen especially for the first peak.

The oscillations following these peaks (especially around $20 \mathrm{eV}$ and above) correspond to the beginning of the EXAFS signal and photoelectron transitions to the continuum state. These oscillations are very distinctive for tetragonal phase. They can be used as a clear fingerprint of the tetragonal phase while the differences between monoclinic and orthorhombic phases are not significant in this part of spectra.

To discriminate effects caused by chemical substitution from structural changes, we also calculated the $\mathrm{O}$ K-edge XANES for the monoclinic phase of $\mathrm{Hf}_{1-\mathrm{x}} \mathrm{Zr}_{\mathrm{x}} \mathrm{O}_{2}$ compounds with $\mathrm{x}=0,0.25,0.5,0.75,1$ (Figure $3 \mathrm{~b}$ ). Here, we see a pure chemical effect as structure remained the same for all $\mathrm{Zr}$ concentrations. This chemical effect manifests itself mainly in a shift of the second peak toward lower energies with increasing of $\mathrm{Zr}$ content. This is due to the energy difference of the $\mathrm{Zr} 4 \mathrm{~d}$ and $\mathrm{Hf} 5 \mathrm{~d}$ (the energy gap between $\mathrm{e}_{\mathrm{g}}$ and $\mathrm{t}_{2 \mathrm{~g}}$ states is smaller for $\mathrm{ZrO}_{2}$ compared to $\mathrm{HfO}_{2}$ ). But the relative intensities and shape of the features does not vary as much as for a change of structure in Figure 3a. Only a small decrease of the amplitude for the second peak is observed. Also, the post-XANES oscillations above $10 \mathrm{eV}$ are very close (slightly moving to lower energies with increase of $\mathrm{Zr}$ concentration) as expected because the structure is the same and only the $\mathrm{Zr}$ concentration changes.

\section{EXPERIMENTAL}

Powders were prepared to obtain reference phases for the most relevant $\mathrm{HfO}_{2}: \mathrm{ZrO}_{2}$ compositions and all accessible polymorphs. Hafnia and zirconia powder samples are both monoclinic. Alloys of these compounds in bulk form are also monoclinic. Therefore, hafnia-yttria was used to stabilize a cubic powder reference. A tetragonal powder reference, however, could not be achieved.

Pure zirconia and hafnia powders were commercial precursors obtained from Tosoh, (Lot ZY000013P) and ABCR (Lot AB174486), respectively. The 50:50 mixtures of hafnia-zirconia and hafnia-yttria were fabricated using conventional solid-state processing methods by doping $\mathrm{HfO}_{2}$ powder with 50 at $\% \mathrm{Y}$ and 50 at\% $\mathrm{Zr}$. The starting powders used were $\mathrm{HfO}_{2}$ (99.95\% purity), $\mathrm{Y}_{2} \mathrm{O}_{3}$ (99.95\% purity), and $\mathrm{ZrO}_{2}$ (99.70\% purity) purchased from Alfa Aesar. Each of the starting powders was weighed using an analytical balance. $\mathrm{HfO}_{2}$ was combined with the respective secondary powder material $\left(\mathrm{Y}_{2} \mathrm{O}_{3}\right.$ or $\left.\mathrm{ZrO}_{2}\right)$ and the powder mixtures were ball milled using ethanol as a solvent and zirconia milling media. Ball milling was used to decrease the particle size of the powder materials. Once ball milling was completed, the milling media were removed, and the powders were dried in an oven at $\sim 100{ }^{\circ} \mathrm{C}$ for $\sim 24$ hours. The dried powder materials were heat treated at $1500^{\circ} \mathrm{C}$ for 2 hours. XRD analysis of the powders was performed using a PANalytical X'Pert PRO diffractometer equipped with multichannel solid-state detector PIXcel. $\mathrm{Cu}$ anode $\mathrm{X}$-ray tube was operated in the $45 \mathrm{kV} / 40 \mathrm{~mA}$ regime ( $\mathrm{K}_{\alpha 1,2}$ radiation was used to obtain diffraction patterns). Diffraction patterns were recorded from $2 \vartheta=15^{\circ}$ to $90^{\circ}$ with a step size of $0.0263^{\circ}$.

Thin film capacitor stacks consisting of $12 \mathrm{~nm}$ TiN (physical vapor deposition) for top and bottom electrode and 20 $\mathrm{nm}$ of $\mathrm{HfO}_{2}, \mathrm{Hf}_{0.5} \mathrm{Zr}_{0.5} \mathrm{O}_{2}, \mathrm{Hf}_{0.25} \mathrm{Zr}_{0.75} \mathrm{O}_{2}$ or $\mathrm{ZrO}_{2}$ (atomic layer deposition - ALD) were deposited on $125 \mu \mathrm{m}$ thick rigid graphite obtained from Goodfellows (product number $\mathrm{C}$ 000400) and, as a reference for electrical characterization, also on Si substrates. Details of the procedure can be found elsewhere ${ }^{76}$. The capacitor stacks were annealed at $600{ }^{\circ} \mathrm{C}$ for $20 \mathrm{~s}$ in $\mathrm{N}_{2}$ atmosphere. On the silicon-based references, $10 \mathrm{~nm}$ Ti as adhesion layer and $30 \mathrm{~nm} \mathrm{Pt}$ were deposited through a shadow mask by electron beam evaporation to form circular contact pads of $200 \mu \mathrm{m}$ diameter. These pads served as hard mask for the subsequent wetchemical removal of the TiN top electrodes in a mixture of $\mathrm{NH}_{4} \mathrm{OH}, \mathrm{H}_{2} \mathrm{O}_{2}$ and $\mathrm{H}_{2} \mathrm{O}(1: 2: 50)$ at $50{ }^{\circ} \mathrm{C}$. Polarization hysteresis of these samples was measured using 
an aixACCT TF Analyzer 3000 with triangular field sweeps of $1 \mathrm{kHz}$ frequency. Grazing incidence X-ray diffraction (GIXRD) was performed on all thin film samples using a Bruker D8 Discover with $\mathrm{Cu}-\mathrm{K}_{\alpha}$ radiation (wavelengths: $0.154 \mathrm{~nm})$.

X-ray absorption spectra of bulk reference and multi-stack thin film samples have been measured at PETRA III, beamline P-65 at DESY, Hamburg. The powders were mixed with cellulose and pressed into pellets. The thin films on carbon were cut to pieces of $5 \mathrm{~mm}$ by $5 \mathrm{~mm}$ and 16 of them stacked between two layers of Kapton tape. Up to four of these packages were put into the $X$-ray to guarantee a reasonable absorption jump close to one. Three

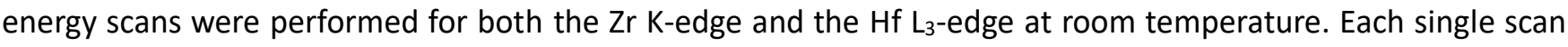
required $180 \mathrm{~s}$ and covered the energy range from $150 \mathrm{eV}$ below the edge to $1000 \mathrm{eV}$ above the edge, as the natural oscillation damping due to thermal and static disorder effects and signal noise in our experimental setup does not allow to obtain longer signal. X-rays were generated by an undulator and monochromatized by a double crystal monochromator using the $\mathrm{Si}(111)$ crystal for the $\mathrm{Hf} \mathrm{L}_{3}$-edge (9561 eV) and the $\mathrm{Si}(311)$ crystal for the $\mathrm{Zr} \mathrm{K}$ edge (17998 eV). The two energy scales were calibrated using a $\mathrm{Hf}$ and a $\mathrm{Zr}$ foils. Higher harmonics were suppressed by using two plane mirrors, $\mathrm{Si} / \mathrm{Si}\left(2 \mathrm{mrad}\right.$ ) in case of the $\mathrm{Hf}_{3}$ - and $\mathrm{Rh} / \mathrm{Rh}$ ( $2 \mathrm{mrad}$ ) in case of the $\mathrm{Zr} \mathrm{K}$-edge. The XAFS spectra were recorded in transmission mode using three ionization chambers with proper gas mixtures to measure the incoming intensity, and the intensities after the sample and the reference foil located in between the second and the third ionization chambers.

Oxygen K-edge XANES spectra were recorded at room temperature in fluorescence mode at the plane grating monochromator (PGM) beamline ${ }^{77}$ of PTB at the BESSY II electron storage ring. This undulator beamline provides soft X-ray radiation of high spectral purity and high radiant power in the photon energy range of $78 \mathrm{eV}$ to $1860 \mathrm{eV}$. Depending on the operational parameters, stray light contributions of about $0.5 \%$ to $1 \%$ have to be taken into account. The uncertainty of the energy scale of the PGM is in the $10^{-4}$ range. For the calibration of the PGM energy scale, typical resonance lines of $\mathrm{Kr}$, Ar and Ne gases are used ${ }^{78}$.

The experiment was carried out using an in-house developed ultrahigh vacuum chamber ${ }^{79}$. The samples were excited using an incident angle of $45^{\circ}$ to suppress any attenuation effects and the emitted oxygen $\mathrm{K}_{\alpha}$ fluorescence radiation was detected using a calibrated silicon drift detector under an angle of $45^{\circ}$. For normalization purposes, the photon energy dependent incident flux of the beamline was measured before using a calibrated photodiode.

X-ray absorption measurements over Si: $\mathrm{HfO}_{2}$ thin films were performed at CLAESS beamline ${ }^{80}$ at the ALBA synchrotron facility. A wiggler source was monochromatized by means of a Si(111) double crystal monochromator, while Rh-coated mirrors were exploited to reject higher harmonics. The spectra were recorded at room temperature in fluorescence mode by means of a single channel silicon drift detector. Several scans were acquired to ensure spectral reproducibility and good signal-to-noise ratio.

All X-ray absorption spectra were extracted and processed using the standard approach ${ }^{54}$ and the software Athena ${ }^{81}$. Fourier transformations were performed using a Kaiser-Bessel window function and a k-space range of $3-13 \AA^{-1}$.

\section{RESULTS AND DISCUSSION}

First, the results on powder reference samples will be shown and explained. After that, the characterization of the thin film capacitor samples is presented and discussed for consistency between electrical and structural data as well as in comparison to similarities or differences with the powder references.

\subsection{Powder References}

Rietveld refinements of the XRD data were performed with the software Profex ${ }^{82}$. The refined diffraction pattern of the undoped hafnia powder is exemplarily shown in Figure 4 while the other refinement curves can be found in the Supporting Information S1 together with the unit cell parameters. Rietveld refinements of the diffraction patterns of both $\mathrm{ZrO}_{2}$ and $\mathrm{Hf}_{0.5} \mathrm{Zr}_{0.5} \mathrm{O}_{2}$ powder showed that both are single-phase monoclinic. For the pure $\mathrm{HfO}_{2}$ powder, the refinement yields a mixture of monoclinic and tetragonal phases (see inset of Figure 4). The lattice 
constants of the monoclinic $\mathrm{Hf}_{1-x} \mathrm{Zr}_{x} \mathrm{O}_{2}$ phase increase with increasing $\mathrm{Zr}$ content. The diffraction pattern of the $\mathrm{Hf}_{0.5} \mathrm{Y}_{0.5} \mathrm{O}_{2-x}$ sample revealed a small amount of $\mathrm{Y}_{2} \mathrm{O}_{3}$ which is not incorporated in hafnia structure and two cubic $\mathrm{Hf}_{0.5} \mathrm{Y}_{0.5} \mathrm{O}_{2-x}$ phases. Both cubic phases can be described with the same space group $\mathrm{Fm} \overline{3} \mathrm{~m}$, but with slightly different lattice parameters of $5.22 \AA$ and $5.16 \AA$. Large peak broadening and peak overlapping does not allow determination of the crystallite sizes of the cubic phases. The final weighted profile $R$-factor $\left(R_{w p}\right)$ is close to the expected $R$-factor ( $R_{\text {exp }}$ - best possible $R_{w p}$ for given data set) in every refinement. Some larger discrepancies between calculated diffraction pattern and the experimental one are observed for the $\mathrm{Hf}_{0.5} \mathrm{Y}_{0.5} \mathrm{O}_{2-x}$ sample, which is indicated by a lower goodness of fit value ${ }^{83}-\chi^{2}=\left(R_{w p} / R_{\text {exp }}\right)^{2}$ than for the other powders (see Supporting Information S1). Table 1 summarizes the results. A stabilization of the tetragonal polymorph in hafnia/zirconia based powders at ambient conditions is unlikely, which is a consequence of their phase diagram with a tetragonal phase only occurring above $1000{ }^{\circ} \mathrm{C} .{ }^{84}$ Therefore, we rely on unit cell data from ab-initio simulation by Materlik et al. ${ }^{39}$ as depicted in Figure 1. These ab-initio results were reported to be in good agreement with the temperature dependent phase diagrams of hafnia and zirconia bulk materials.

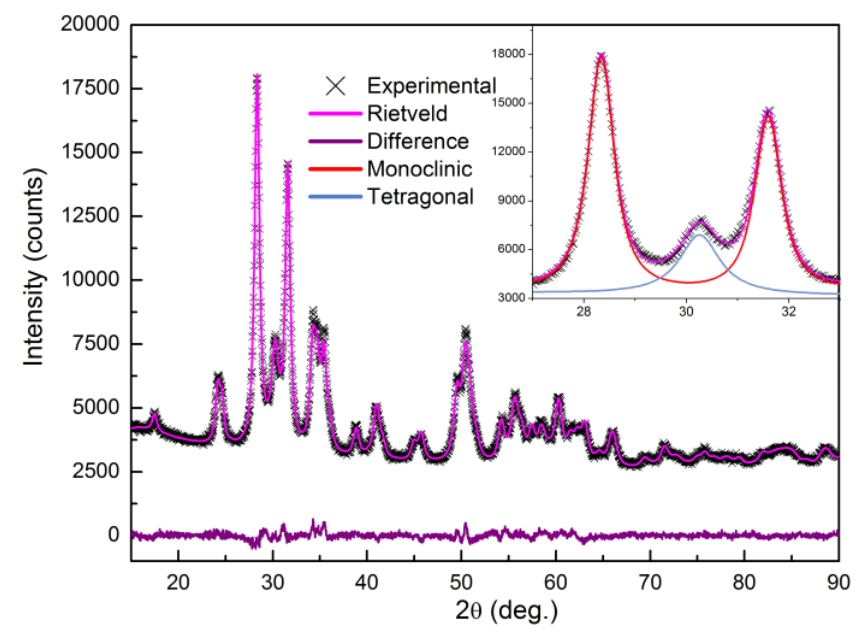

Figure 4. Example of X-ray diffraction pattern and Rietveld fit of the undoped $\mathrm{HfO}_{2}$ powder. The inset shows the magnified region around $2 \vartheta=30^{\circ}$ with separated reflections of the monoclinic and tetragonal phases.

Table 1. Results of Rietveld refinements of the diffraction patterns for the bulk reference samples.

\begin{tabular}{|c|c|c|c|c|c|}
\hline Composition & $\begin{array}{c}\text { Phases } \\
\text { (wt\%) }\end{array}$ & $\begin{array}{l}a \\
(\AA)\end{array}$ & $\begin{array}{l}\mathrm{b} \\
(\AA ̊)\end{array}$ & $\begin{array}{l}\mathrm{c} \\
(\AA)\end{array}$ & $\begin{array}{l}\beta \\
\left({ }^{\circ}\right)\end{array}$ \\
\hline $\mathrm{ZrO}_{2}$ & $\begin{array}{c}100 \\
\text { monocl. }\end{array}$ & 5.148 & 5.211 & 5.313 & 99.19 \\
\hline $\mathrm{Hf}_{0.5} \mathrm{Zr}_{0.5} \mathrm{O}_{2}$ & $\begin{array}{c}100 \\
\text { monocl. }\end{array}$ & 5.135 & 5.194 & 5.306 & 99.25 \\
\hline \multirow{2}{*}{$\mathrm{HfO}_{2}$} & $\begin{array}{c}82 \pm 1 \\
\text { monocl. }\end{array}$ & 5.131 & 5.172 & 5.305 & 99.11 \\
\hline & $\begin{array}{l}18 \pm 1 \\
\text { tetrag. }\end{array}$ & \multicolumn{2}{|c|}{3.593} & 5.185 & 90 \\
\hline \multirow{3}{*}{$\mathrm{Hf}_{0.5} \mathrm{Y}_{0.5} \mathrm{O}_{2-\mathrm{x}}$} & $\begin{array}{l}7 \pm 1 \\
\mathrm{Y}_{2} \mathrm{O}_{3}\end{array}$ & \multicolumn{3}{|c|}{10.599} & 90 \\
\hline & $\begin{array}{l}56 \pm 1 \\
\text { cubic I }\end{array}$ & \multicolumn{3}{|c|}{5.221} & 90 \\
\hline & $\begin{array}{c}37 \pm 1 \\
\text { cubic II }\end{array}$ & \multicolumn{3}{|c|}{5.164} & 90 \\
\hline
\end{tabular}


The $\mathrm{Hf}_{3}$-edge $\mathrm{X}$-ray absorption near edge structure (XANES) spectra of the $\mathrm{HfO}_{2}, \mathrm{Hf}_{0.5} \mathrm{Zr}_{0.5} \mathrm{O}_{2}$ and $\mathrm{Hf}_{0.5} \mathrm{Y}_{0.5} \mathrm{O}_{2-\mathrm{x}}$ powders show trends consistent with the structure data, but do not allow for deeper insights with respect to the structure. It appears that electronically, the samples have very similar electronic structure. A comparison and related discussions can be found in in the Supporting Information S2.

The EXAFS parts of the spectra exhibit distinct features for the pure $\mathrm{HfO}_{2}, \mathrm{Hf}_{0.5} \mathrm{Zr}_{0.5} \mathrm{O}_{2}$ and $\mathrm{Hf}_{0.5} \mathrm{Y}_{0.5} \mathrm{O}_{2-x}$ powders as shown in the FT-EXAFS in Figure 5. The corresponding EXAFS spectra are presented in Supporting Information S5.

The overall impression is very straightforward: the local structure of Zr for the bulk samples does not change a lot, as all features in the Zr K-edge EXAFS spectra and corresponding FT-EXAFS are the same within range of our spectra (Figure 5b). This is in line with XRD data where $\mathrm{Hf}_{0.5} \mathrm{Zr}_{0.5} \mathrm{O}_{2}$ and the $\mathrm{ZrO}_{2}$ look to be $100 \%$ monoclinic. The first coordination shell $(\mathrm{Zr}-\mathrm{O})$ has the least changes indicating that $\mathrm{ZrO}_{7}$ polyhedra retain the same statistics (combination of distances and their variation) which is expected as both compounds have the same monoclinic symmetry.

At the same time, the decrease in FT-EXAFS amplitude for second coordination shell could be correlated to the mixture of the atoms in the second coordination shell ( $\mathrm{Zr}-\mathrm{Me}$, where $\mathrm{Me}=\mathrm{Zr}, \mathrm{Hf}$ ). However, our simulations suggest that this feature should be significantly lower and more diffuse (see Figure 2 (b) for $50 \%$ of Zr). This might indicate that the second coordination shell of $\mathrm{Zr}$ might have higher concentration of $\mathrm{Zr}$ atoms than we expect in the case of a random distribution. We see this as a sign of $Z r$ segregation in some small clusters (can be even just a higher than a statistically averaged concentration of $\mathrm{Zr}-\mathrm{O}-\mathrm{Zr}$ groups compared to $\mathrm{Zr}$-O-Hf ones).

The local structure of $\mathrm{Hf}$ represented by the $\mathrm{Hf}_{3}$-edge EXAFS and FT-EXAFS behaves close to the theoretical calculations for monoclinic structure. The FT-EXAFS features corresponding to the first shell (Hf-O) are very close, especially for $\mathrm{HfO}_{2}$ and $\mathrm{Hf}_{0.5} \mathrm{Zr}_{0.5} \mathrm{O}_{2}$ as in $\mathrm{HfO}_{2}$, the monoclinic phase dominates. This is supported by our theoretical

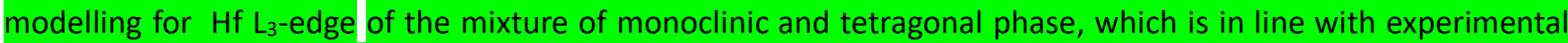
spectra (Figure 5a, Figure S16b in Supporting Information) where in both we see a single peak for Hf-O and double peak for Hf-Hf coordination shells.

The slight increase of the maximum amplitude of the $\mathrm{Hf}-\mathrm{O}$ feature in FT-EXAFS for $\mathrm{Hf}_{0.5} \mathrm{Y}_{0.5} \mathrm{O}_{2-\mathrm{x}}$ (Figure $5 \mathrm{a}$ ) is expected due to a narrower distance distribution in the cubic phase, but the difference should be much bigger (Figure $1 b)$ if we had a single pure cubic phase instead of two as detected by XRD. 
Bulk Reference Samples
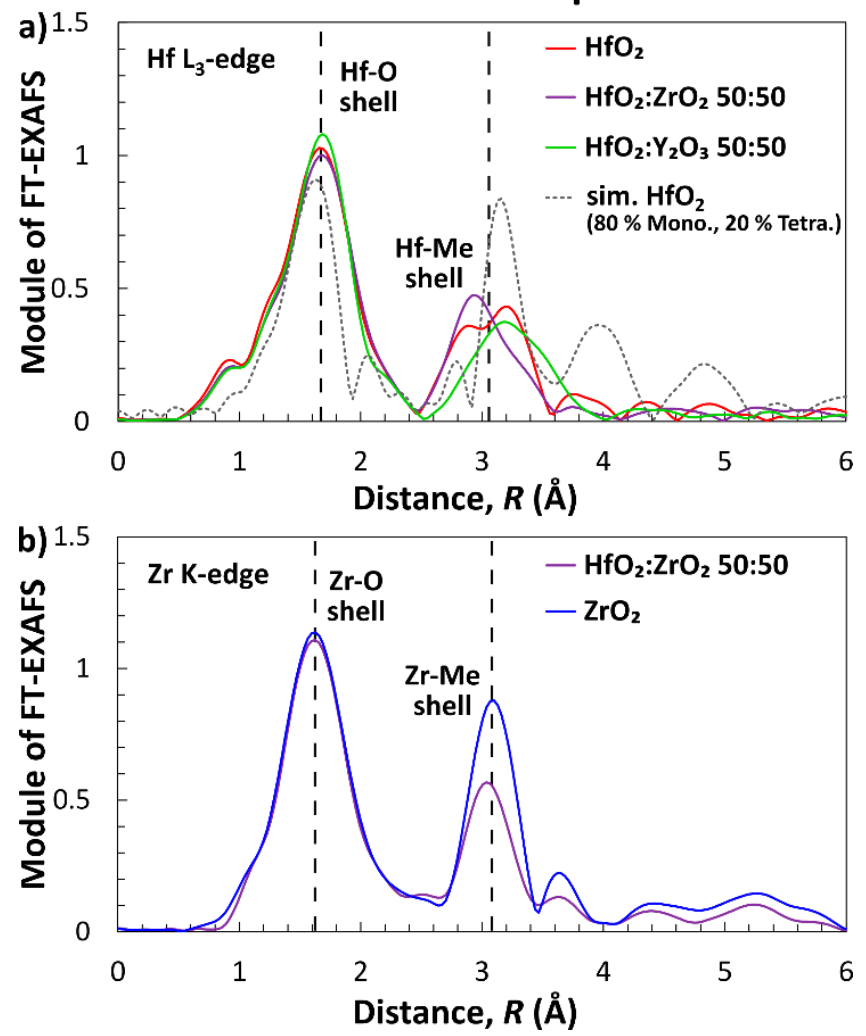

Figure 5. (a) $\mathrm{Hf} \mathrm{L}_{3}$-edge and (b) $\mathrm{Zr} \mathrm{K}$-edge FT-EXAFS for $\mathrm{HfO}_{2}, \mathrm{Hf}_{0.5} \mathrm{Zr}_{0.5} \mathrm{O}_{2}, \mathrm{Hf}_{0.5} \mathrm{Y}_{0.5} \mathrm{O}_{2-y}, \mathrm{ZrO}_{2}$ powders. For the $\mathrm{Hf} \mathrm{L}_{3^{-}}$ edge a theoretical spectrum of a mixture of $80 \%$ monoclinic and $20 \%$ tetragonal phase is provided (Please note that the amplitudes at higher $R$ values are more pronounced as explained in the beginning of section 2). The vertical dashed lines are provided just for guidance to highlight the changes between the main peaks of the Me-O and Me-Me coordination shells. Note that the distances do not correspond to the real distances due to phase shift of the photoelectron. Distances for Hf and Zr are not comparable as this phase shift is different for Hf and Zr. However, the distances of the respective $\mathrm{Hf}$ or $\mathrm{Zr}$ edges can be compared between Figure 5 and Figure 9.

The changes in the second coordination shell of $\mathrm{Hf}(\mathrm{Hf}-\mathrm{Me})$ with the composition are more pronounced, so it contains more useful information to study composition and structure evaluation than the first coordination shell. Comparing the FT-EXAFS features corresponding to the second coordination shell of $\mathrm{Hf}$ for $\mathrm{HfO}_{2}$ and $\mathrm{Hf}_{0.5} \mathrm{Zr}_{0.5} \mathrm{O}_{2}$ it can be seen that the major difference is the feature at 3.3 $\AA$. This feature is close to the maximum of the corresponding feature for cubic phase and calculated tetragonal phase. Here, the EXAFS data support the XRD finding that this is a mixture of monoclinic and tetragonal phase as our theoretical spectra for monoclinic and tetragonal phase mixture match the experimental one (Figure 5a). The maximum of the $\mathrm{Hf}-\mathrm{Me}$ feature for $\mathrm{Hf}_{0.5} \mathrm{Zr}_{0.5} \mathrm{O}_{2}$ moves to shorter distances slightly below $3 \AA$ because of two effects: The tetragonal phase disappears because this sample has a pure monoclinic phase. Moreover, adding $50 \%$ of $\mathrm{Zr}$ shifts the feature of the second shell to shorter distances and changes shape as the theoretical calculations in Figure 2 show. We can confirm the findings form the $\mathrm{Zr} \mathrm{K}$-edge data: The amplitude of the Hf-Me signal does not correspond to $50 \% \mathrm{Zr}$ but is somewhere between $25 \%$ and $50 \% \mathrm{Zr}$. This shows that the concentration of $\mathrm{Zr}$ in the second coordination shell of Hf is lower than expected from an even distribution of $\mathrm{Zr}$ and $\mathrm{Hf}$ atoms. This finding is based on a comparison of the relative amplitudes for the model spectra for hafnia with 80:20 mixture of monoclinic and tetragonal phase with model spectra for $\mathrm{Hf}_{0.5} \mathrm{Zr}_{0.5} \mathrm{O}_{2}$ and $\mathrm{Hf}_{0.75} \mathrm{Zr}_{0.25} \mathrm{O}_{2}$ (Figure S18 in the Supporting Information) with the proportions in the experimental spectra for $\mathrm{HfO}_{2}$ and $\mathrm{Hf}_{0.5} \mathrm{Zr}_{0.5} \mathrm{O}_{2}$ powders. 
Comparing $\mathrm{Zr} \mathrm{K}$-edge and $\mathrm{Hf}_{3}$-edge spectra for $\mathrm{HfO}_{2}, \mathrm{Hf}_{0.5} \mathrm{Zr}_{0.5} \mathrm{O}_{2}$ and $\mathrm{ZrO}_{2}$, we can conclude that for the $\mathrm{Hf}_{0.5} \mathrm{Zr}_{0.5} \mathrm{O}_{2}$ bulk material, the local structure of $\mathrm{Zr}$ and $\mathrm{Hf}$ does not correspond to an even distribution of $\mathrm{Zr}$ and $\mathrm{Hf}$ atoms on the lattice sites of the unit cells (Figure 2 and Figure 5). This is a sign of nano-segregation of having more $\mathrm{Zr}$ in the second coordination shell of $\mathrm{Zr}$ and more $\mathrm{Hf}$ in the second coordination shell of $\mathrm{Hf}$ than expected from statistically even distribution. It should be noted that no ordering of $\mathrm{Hf}$ and $\mathrm{Zr}$ atoms in any superstructures or a phase separation were detected in XRD measurements.

\subsection{Thin Film Capacitor Stacks}

Figure 6 shows the basic electrical and structural characterization of the chosen hafnia-zirconia compositions between TiN electrodes on Si substrates. As expected from previous works ${ }^{76,85}$, ALD-deposited pure hafnia exhibits a monoclinic phase in the grazing incidence X-ray diffraction (GIXRD) patterns (Figure 6b), related with linear dielectric behavior (Figure 6a). Figure 6, panels b and c, compares the GIXRD patterns collected for different zirconia contents for film stacks on a $\mathrm{Si}$ and on a rigid graphite substrates. The latter were used for the X-ray absorption spectroscopy experiments. For increasing zirconia content, hysteretic curves appear. For the 50:50 mixture, a ferroelectric hysteresis is seen, which is related with the orthorhombic phase, but not phase-pure as the GIXRD pattern suggests. A significant amount of monoclinic phase is present in the film and even the existence of tetragonal grains cannot be ruled out from these patterns with broad and overlapping peaks. However, as the tetragonal phase gives rise to antiferroelectric properties and a pinched hysteresis like for the $\mathrm{Hf}_{0.25} \mathrm{Zr}_{0.75} \mathrm{O}_{2}$ and the $\mathrm{ZrO}_{2}$ films, significant portions of tetragonal phase are unlikely. Comparing the GIXRD results on $\mathrm{Si}$ with those on $\mathrm{C}$ substrate as used for the $\mathrm{x}$-ray absorption measurements, the $\mathrm{Hf}_{0.5} \mathrm{Zr}_{0.5} \mathrm{O}_{2}$ samples exhibits even higher monoclinic peaks making the presence of a tetragonal phase even less likely if we rely on the general trends explained by Hoffmann et al. ${ }^{18}$. For even higher $\mathrm{Zr}$ content, antiferroelectric behavior and a tetragonal phase are observed. Due to the brittle constitution of the graphite substrate, electrical measurements were not possible. The striking similarity of their GIXRD patterns (Figure $6 c$ ) to those of the reference film stacks on silicon allows us to conclude on the presence of comparable phase fractions and thus, comparable dielectric behavior. Just a slightly trend toward more monoclinic phase fractions can be concluded from the comparison of Figure $6 \mathrm{~b}$ and $\mathrm{c}$. With these vital prerequisites met, we can proceed to the discussion of the EXAFS measurements below.

The $\mathrm{Hf}_{2}$-edge EXAFS obtained from a single $\mathrm{Si} \mathrm{HfO}_{2}\left(1\right.$ at\%, ratio of $\left.\mathrm{Si} /[\mathrm{Hf}+\mathrm{Si}+\mathrm{O}]^{45}\right)$ thin film deposited on a 675 $\mu \mathrm{m}$ thick Si substrate obtained in a preliminary fluorescence mode measurement at the CLAESS beamline at the ALBA synchrotron facility is compared to the signal recorded using the multi-stack approach for a pure $\mathrm{HfO}_{2}$ film in Figure 7 (details and full data, see Supporting Information S4). Both signals are similar, as they both correspond to the monoclinic phase. However, there is an obvious difference in the quality of the EXAFS data, especially for values of the wavenumber $k$ above $8 \AA^{-1}$. The quality of the signal obtained from stacking multiple thin film capacitors on the thin carbon substrate is comparable with that of the bulk references (see FT-EXAFS in Figure 5 and EXAFS data in Figures S8 and S9 in the Supporting Information). This clearly proves the advantages of this approach compared to measuring a single hafnia film in fluorescence mode as it has been done previously by the authors and other groups ${ }^{86}$.

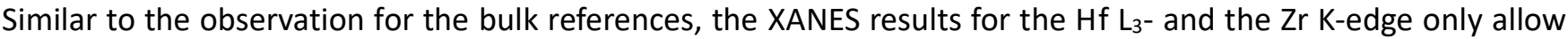
limited conclusions. However, the trends are more pronounced then for the bulk samples as the changes in phase composition are more significant in the thin films. A more detailed discussion of the spectra can be found in the Supporting Information S3.

XANES measurements at a soft X-ray beamline, the PGM beamline ${ }^{77}$ of the PTB at BESSY II were performed to check whether the sensitivity of the $\mathrm{O}$ K-edge is sufficient to see marked changes in the spectra. The $\mathrm{O}$ K-edge XANES spectra reported in Figure 8 show significant differences. The spectra consist of two main features around $532 \mathrm{eV}$ and $536 \mathrm{eV}$, corresponding to the empty $\mathrm{e}_{\mathrm{g}}$ and $\mathrm{t}_{2 \mathrm{~g}}$ states of $\mathrm{Hf} 5 \mathrm{~d}$ or $\mathrm{Zr} 4 \mathrm{~d}$, followed by a broader and more complex peak sequence starting at $540 \mathrm{eV}$, which originates from $\mathrm{Hf} 6 \mathrm{sp}$ and $\mathrm{Zr} 5 \mathrm{sp}$ empty hybrid states, hybridized with the $\mathrm{O} 2 \mathrm{p}$ bands ${ }^{58,61,63}$. The shoulder at $531 \mathrm{eV}$ most likely stems from the interface states (probably Ti-O as all thin films are sandwiched between TiN layers ${ }^{87}$, or a $\mathrm{C}=\mathrm{O}$ signal from carbon substrate). 

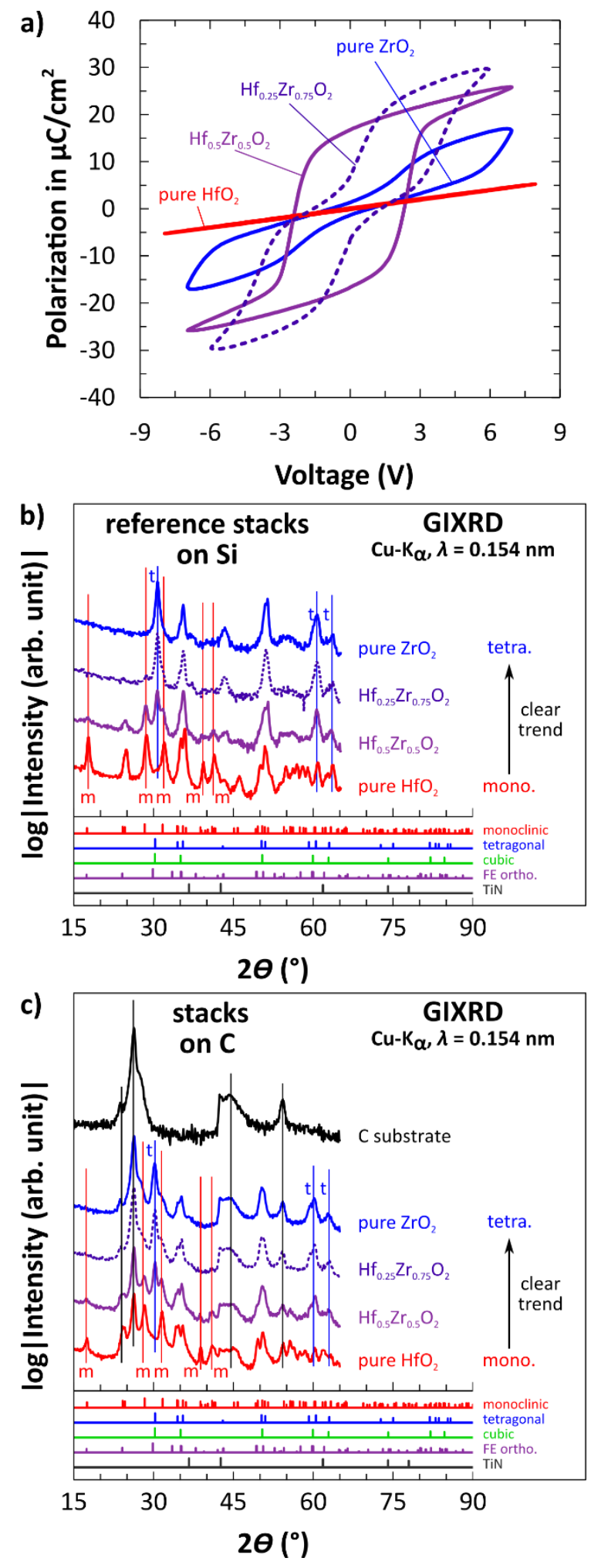

Figure 6. (a) Polarization vs. electric field curves from dynamic hysteresis measurements of the hafnia-zirconia based films and (b) corresponding grazing incidence X-ray diffractograms for the different zirconia contents for reference stacks on a Si substrate as the base of the observed dielectric behavior. (c) Grazing incidence X-ray diffractograms of the film stacks on rigid graphite substrates that were used for the X-ray absorption spectroscopy experiments. 
The O K-edge XANES data give more profound information about structural transformations (compared to the Hf $\mathrm{L}_{3}$-edge or Zr K-edge) and support a transformation of the spectra from monoclinic toward tetragonal as seen for the post-XANES oscillations, e.g. around $555 \mathrm{eV}$. These changes are in line with theoretical calculations presented in Figure 3. For $75 \% \mathrm{ZrO}_{2}$ content, the electronic local structure for oxygen looks almost identical to the one of pure $\mathrm{ZrO}_{2}$.

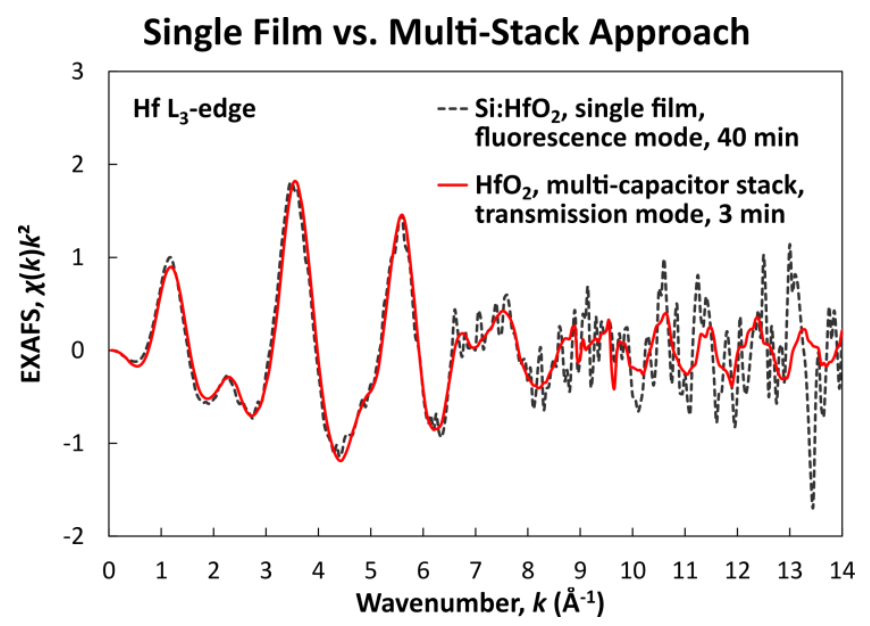

Figure 7. $\mathrm{Hf}_{3}$-edge, EXAFS spectra for a single Si:HfO ${ }_{2}$ thin film on Si in fluorescence mode and multiple stacks of a $\mathrm{HFO}_{2}$ film on carbon in transmission mode.

Moreover, it can be seen that the $\mathrm{Hf}_{0.5} \mathrm{Zr}_{0.5} \mathrm{O}_{2}$ thin films show noticeable differences to monoclinic structure of $\mathrm{HfO}_{2}$ thin films in this region above $545 \mathrm{eV}$. However, this is most likely due to the impact of substituting $\mathrm{Hf}$ with $\mathrm{Zr}$ as our theoretical modelling indicates. The differences between monoclinic and orthorhombic structures in Figure 3 are small compared to changes found for chemical substitution.

The shift of the $t_{2 g}$ peak to lower energies (decreasing gap between $e_{g}$ and $t_{2 g}$ states) is also caused mainly by the substitution of $\mathrm{Hf}$ with $\mathrm{Zr}$ as explained in the simulations section. The sharpening of these features, on the contrary, is a signature of structural transformations (especially the sharpening of the first peak) as found in our theoretical calculations. It should be noted that the simulated spectra for the monoclinic phase do not reproduce the experimental spectra very well. The simulated spectra exhibit much broader and less defined features at $533 \mathrm{eV}$ than the experimental ones. This can be due to issues with the self-consistent calculations of the XANES spectra leading to different positions of the Fermi level for different absorbers or a too broad distribution of Me-O distances in the model (as compared to reality). Both of these effects might lead to broader spectral features. Nonetheless, the predicted trends of the transformation of the XANES spectra are well aligned: 1) From a monoclinic to a tetragonal phase, a broader multi-peak structure transforms into a two-peak structure. 2) From $\mathrm{HfO}_{2}$ to $\mathrm{ZrO}_{2}$, the features shift to lower energies.

So, in the $\mathrm{O}$ K-edge as well as in the $\mathrm{Zr}$ K-edge and $\mathrm{Hf} \mathrm{L}_{3}$-edge XANES spectra (see Supporting Information S3), we can consistently observe a gradual transformation from monoclinic to tetragonal phase as the $\mathrm{Zr}$ content of the films increases. 


\section{Multi-Stack Thin Film Samples}

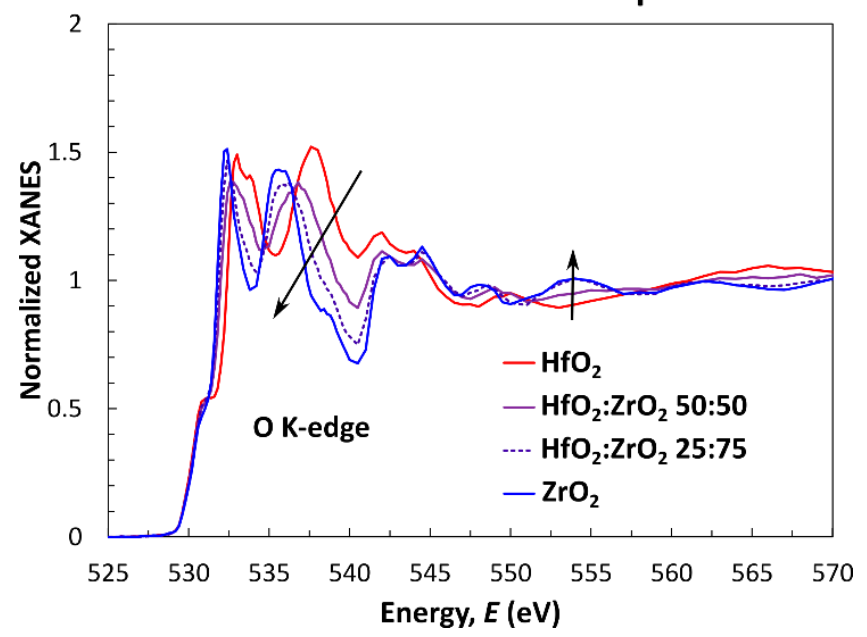

Figure 8. O K-edge XANES spectra for $\mathrm{Hf}_{1-\mathrm{x}} \mathrm{Z} \mathrm{r}_{x} \mathrm{O}_{2}$ thin films showing gradual transformation of the spectra from monoclinic (pure $\mathrm{HfO}_{2}$ thin film) to tetragonal phase (pure $\mathrm{ZrO}_{2}$ thin film). The arrow indicates the trend of the

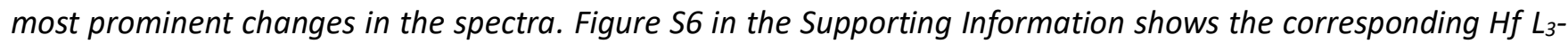
and Zr K-edge spectra.

A comparison of the FT-EXAFS spectra obtained on the $\mathrm{Hf} \mathrm{L}_{3}$ and $\mathrm{Zr}$ K-edge for $\mathrm{Hf}_{1-\mathrm{x}} \mathrm{Zr}_{\mathrm{x}} \mathrm{O}_{2}$ multi-stack thin films samples is reported in Figure 9 (see Figure S9 in the Supporting Information for the corresponding EXAFS spectra). Comparing the results to those in Figure 5, it can be concluded that the local structure of $\mathrm{Hf}$ in the pure $\mathrm{HfO}_{2}$ thin films is close to that of the bulk samples, while for the $\mathrm{ZrO}_{2}$, bulk and thin film spectra are significantly differentthe former being related to a monoclinic the latter to a tetragonal phase.

The close similarity of $\mathrm{HfO}_{2}$ thin film, the powder reference and theoretical spectra for pure monoclinic phase suggests that the monoclinic phase is dominant. While the powder contains $80 \%$ of the tetragonal phase, the GIXRD pattern of the thin film (Figure 6) does not show any noticeable traces of the tetragonal phase. Another simple explanation for why the EXAFS spectra still look very similar (beyond the dominating monoclinic phase mentioned before) is that if fractions of the tetragonal phase might be present in the thin films, the size of the corresponding crystallites is much smaller resulting in a very weak contribution to the GIXRD data.

The $\mathrm{Hf}_{3}$-edge spectra of the ferroelectric $\mathrm{Hf}_{0.5} \mathrm{Zr}_{0.5} \mathrm{O}_{2}$ thin film shares some similarity with the one of monoclinic bulk $\mathrm{Hf}_{0.5} \mathrm{Zr}_{0.5} \mathrm{O}_{2}$, but the signal from the second coordination shell $\mathrm{Hf}-\mathrm{Me}$ exhibits some differences: It is narrower and slightly shifted to shorter distances compared to bulk (Figures S9-S10 in the Supporting Information). If we compare this to our calculations for the substitution of $\mathrm{Hf}$ with $\mathrm{Zr}$ (Figure 2), we see that this corresponds exactly to the case of $50 \% \mathrm{Zr}$ where the peak of the $\mathrm{Hf}-\mathrm{Me}$ feature shifts to shorter distances and becomes narrower.

At the same time, the $\mathrm{Zr}$ K-edge FT-EXAFS spectrum for the same $\mathrm{Hf}_{0.5} \mathrm{Zr}_{0.5} \mathrm{O}_{2}$ thin film is clearly different from that of the powder reference with the same composition. The second and further coordination shells are more disordered and do neither match the tetragonal (as for $\mathrm{ZrO}_{2}$ thin films) nor the monoclinic (as for $\mathrm{Hf}_{0.5} \mathrm{Zr}_{0.5} \mathrm{O}_{2}$ bulk samples) reference spectra. However, the thin film data is in excellent agreement with our model calculations for $\mathrm{Hf}_{0.5} \mathrm{Zr}_{0.5} \mathrm{O}_{2}$ with random distribution of $\mathrm{Zr}$ and $\mathrm{Hf}$ in the second (and further) coordination shell of $\mathrm{Zr}$. This together

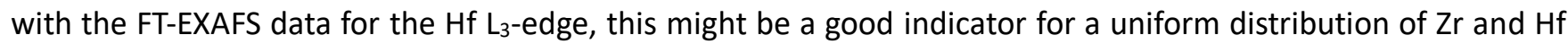
atoms in the films. According to GIXRD and the dielectric measurements in Figure 6, the thin film is expected to be a mixture of mainly monoclinic and orthorhombic phase. This is also in line with the EXAFS data, as differences in the spectra of these two phases are expected to be small (Figure 1a). Altogether, this raises the question, who

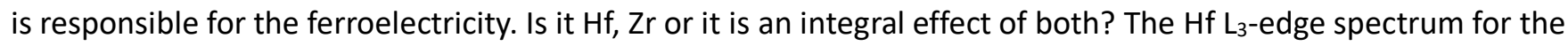
$\mathrm{Hf}_{0.25} \mathrm{Zr}_{0.75} \mathrm{O}_{2}$ looks different from both the spectra of the $\mathrm{Hf}_{0.5} \mathrm{Zr}_{0.5} \mathrm{O}_{2}$ and of the $\mathrm{HfO}_{2}$ thin films indicating not only chemical, but also structural differences. In FT-EXAFS, a double feature is found for the first shell of $\mathrm{Hf}(\mathrm{Hf}-\mathrm{O})$, 
which is characteristic for the tetragonal phase as follows from our theoretical calculations of EXAFS spectra (Figure 1). At the same time, a feature corresponding to the second coordination shell is more disordered, which is in better agreement with the model spectra (Figure 2 as well as Figures S13 and S14) for tetragonal phase with significantly lower $\mathrm{Zr}$ concentration. In the tetragonal $\mathrm{Hf}_{0.25} \mathrm{Zr}_{0.75} \mathrm{O}_{2}$ model compound of the monoclinic phase, the $\mathrm{Hf}$-Me feature is much more pronounced than for lower concentrations of Zr. The same, however, applies also for monoclinic and orthorhombic model systems, where the height of the FT-EXAFS feature for second coordination shell is maximal for the highest $\mathrm{Zr}$ concentrations. This is mainly strongly impacted by a chemical effect, while the first coordination shell reflects mainly the structural effect.
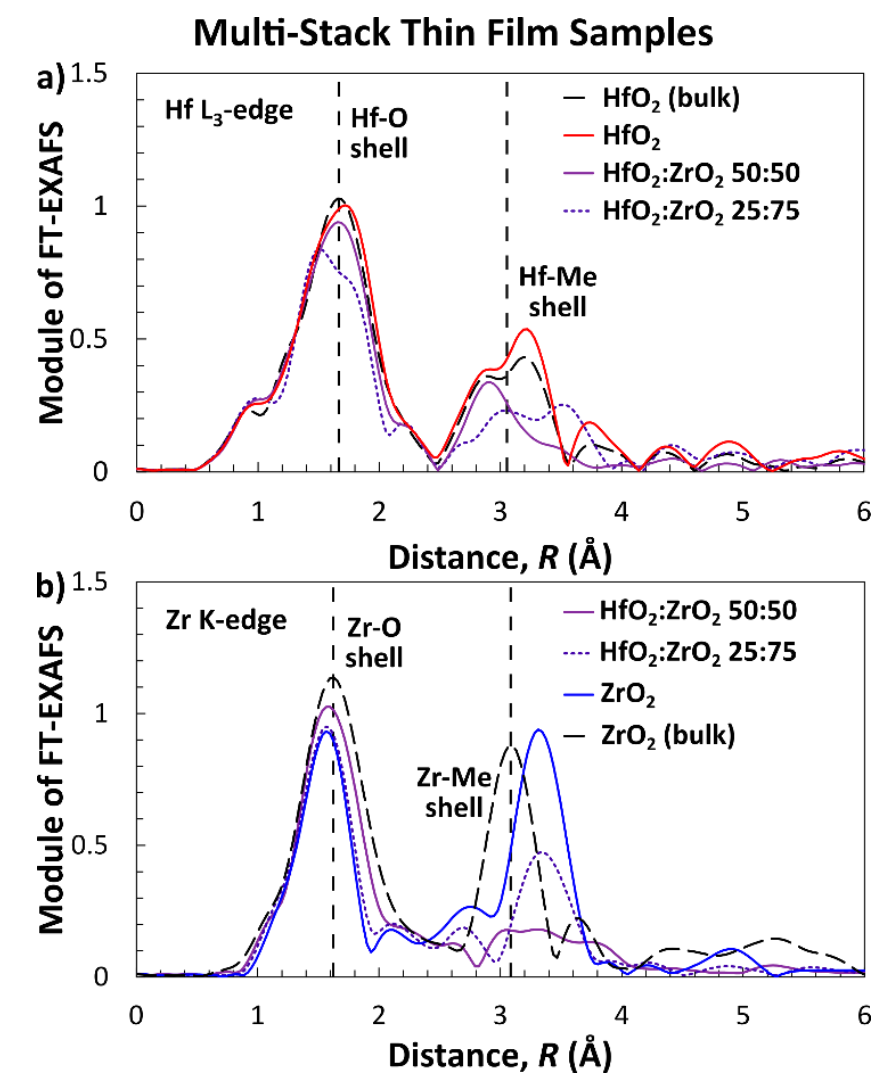

Figure 9. (a) $H f L_{3}$-edge and (b) Zr K-edge FT-EXAFS for $\mathrm{Hf}_{1-\mathrm{x}} \mathrm{Zr}_{x} \mathrm{O}_{2}(x=0,0.5,0.75,1$.) thin films. The vertical dashed lines highlight the shifts between the main peaks of the Me-O and Me-Me shells when comparing the Hf and the $Z r$ edge spectra. Note that the distances do not correspond to the real distances due to phase shift of the photoelectron. Distances for $\mathrm{Hf}$ and $\mathrm{Zr}$ are not comparable as this phase shift different for $\mathrm{Hf}$ and $\mathrm{Zr}$. However, the distances of the respective $\mathrm{Hf}$ or Zr edges can be compared between Figure 5 and Figure 9. Corresponding EXAFS files are shown in Supporting Information Figure S1O.

The $\mathrm{Zr}$ K-edge spectra for $\mathrm{Hf}_{0.25} \mathrm{Zr}_{0.75} \mathrm{O}_{2}$ and $\mathrm{ZrO}_{2}$ thin films show features characteristic for the tetragonal phase. The only difference is found in the amplitude of the spectra, which can be attributed to the chemical disorder from mixing $\mathrm{Zr}$ and $\mathrm{Hf}$ as evidenced by our model calculations (Figure 2). The only remarkable difference is that we do not see the double feature for $\mathrm{Zr}-\mathrm{O}$ in the first coordination shell, which is a fingerprint of the tetragonal phase. This might hint on dynamic correlation effects. The dynamic correlation effects might change the shape of radial distribution function (RDF) ${ }^{88}$. Dielectric and GIXRD measurements (Figure 6) show that these thin films are tetragonal and antiferroelectric. This can be due to a pseudo-symmetry effect: The Me atoms occupy a set of tetragonal positions, which dominate the measured XRD signal. The $O$ atoms might, however be closer to the positions in the cubic unit cell, which would not result in two distinct Me-O distances. 
Given that the orthorhombic phase shares some similarities with the monoclinic phase ${ }^{89}$ and the transition state between the two polarization states has been described as an orthogonalized monoclinic phase ${ }^{90}$, the interpretation data for thin films becomes a complex subject. Moreover, Grimley et al. ${ }^{50}$ showed the coexistence of multiple phases within one grain that relax into one another from one unit cell to another. Future data analysis based on reverse Monte Carlo simulations in combination with neural network approach and spectra acquired at different temperatures will help deciding which of the two explanations is more accurate.

\section{CONCLUSION}

The theoretical EXAFS spectra presented in the beginning of this work show that good data quality is warranted to distinguish the relevant phases in hafnia and zirconia. Differences in the spectra of the monoclinic, orthorhombic, tetragonal and cubic phases are mainly observed in the second and higher coordination shells. Targeting the analysis of polycrystalline, only some tens of nm thick films, we therefore, started with powder samples to obtain reference spectra free of thin film effects. The experimental data of the powder references matched well with the theoretical spectra anticipated from the phase composition extracted from XRD via Rietveld refinement and unit cell data from ab-initio simulations. The XANES features and the position of the absorption edges of the powders look very similar hinting on comparable electronic structure despite the apparent differences between the unit cells (compare images in ref. 39).

For all powder compositions, we found a similar local structure for $\mathrm{Hf}$ and $\mathrm{Zr}$. Our pure $\mathrm{HfO}_{2}$ powder is a mixture of monoclinic and tetragonal phase. This questions the usability of this bulk material as a reference samples.

Only the $\mathrm{Hf}_{0.5} \mathrm{Zr}_{0.5} \mathrm{O}_{2}$ might be used as a reference sample, where both $\mathrm{Hf}$ and $\mathrm{Zr}$ exhibit a monoclinic local structure. But even in this case, comparing $\mathrm{Zr}$ K-edge spectra for $\mathrm{Hf}_{0.5} \mathrm{Zr}_{0.5} \mathrm{O}_{2}$ and $\mathrm{ZrO}_{2}$ with the model calculations, we conclude that for the monoclinic $\mathrm{Hf}_{0.5} \mathrm{Zr}_{0.5} \mathrm{O}_{2}$ bulk material, the local structure of $\mathrm{Zr}$ and $\mathrm{Hf}$ differs from that of a random distribution of $\mathrm{Zr}$ and $\mathrm{Hf}$ atoms because the experimental data (Figure 5) does not agree with the simulations for monoclinic $\mathrm{Hf}_{0.5} \mathrm{Zr}_{0.5} \mathrm{O}_{2}$ (Figure 2). We see signs of an increased probability of $\mathrm{Zr}-\mathrm{O}-\mathrm{Zr}$ and $\mathrm{Hf}-\mathrm{O}-\mathrm{Hf}$ structures compared to a statistically even distribution.

The cubic structure of the $\mathrm{Y}_{2} \mathrm{O}_{3}: \mathrm{HfO}_{2}$ mixture has a clearly different appearance also in the experimental EXAFS spectra of the bulk samples. Features corresponding to the second coordination shell of the metal (here $\mathrm{Hf}$ ) seen in the FT-EXAFS around $3 \AA$ in Figure 5a are not as sharp and pronounced as expected for single cubic phase. This is in line with the Rietveld results that concluded the presence of two cubic hafnia-yttria phases combined with a chemical mixture of $\mathrm{Y}$ and $\mathrm{Hf}$ atoms and including a small presence of $\mathrm{Y}_{2} \mathrm{O}_{3}(7 \%)$. Moreover, it is likely that the non-ideal random distribution of the substitute element into the $\mathrm{Hf}$ sites found for $\mathrm{Hf}_{0.5} \mathrm{Zr}_{0.5} \mathrm{O}_{2}$, also makes the features of the $\mathrm{Y}_{2} \mathrm{O}_{3}: \mathrm{HfO}_{2}$ powder less sharp than in theory due to the static disorder.

So, we can summarize that obtaining good single-phase bulk $\mathrm{Hf}_{1-x} \mathrm{Zr}_{\mathrm{x}} \mathrm{O}_{2}$ and $\mathrm{Hf}_{0.5} \mathrm{Y}_{0.5} \mathrm{O}_{2-\mathrm{x}}$ reference compounds with random distribution of atoms is still an open challenge.

Thin films ranging from paraelectric monoclinic via ferroelectric orthorhombic to antiferroelectric tetragonal were fabricated and analyzed via GIXRD and P-E hysteresis measurements. The simple approach of measuring a single $10 \mathrm{~nm}$ thick film on $\mathrm{Si}$ in fluorescence mode was shown to be insufficient to obtain the required data quality that allows the analysis of higher coordination shells. Therefore, capacitor stacks were fabricated on thinner $C$ substrates and stacked on top of one another. A signal quality comparable to the bulk samples could be achieved.

The trends in XANES and EXAFS could be correlated with GIXRD and P-E measurements as well as with the bulk

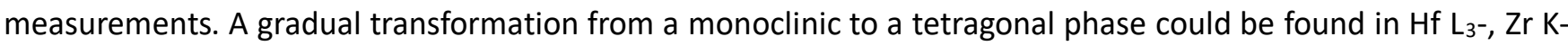
and $O$ K-edge XANES spectra, with most prominent changes found in the latter ones.

The $\mathrm{O}$ K-edge XANES data (Figure 8) give more profound information about structural transformations compared

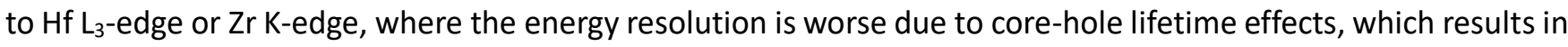
a smoothening of all features. The O K-edge XANES spectra support a transformation of the spectra from monoclinic to tetragonal as is very evident from the post-XANES oscillations (compare Figure 3). With $75 \%$ of $\mathrm{ZrO}_{2}$ content, the electronic local structure for oxygen looks almost identical to the one of pure $\mathrm{ZrO}_{2}$. 
Also, the $\mathrm{Hf}_{0.5} \mathrm{Zr}_{0.5} \mathrm{O}_{2}$ thin films show noticeable differences in this post-XANES (early EXAFS) region compared to monoclinic structure of the $\mathrm{HfO}_{2}$ thin films. This effect, however, is most likely due to the substitution of $\mathrm{Hf}$ with $\mathrm{Zr}$ as indicated by in our theoretical modelling (Figure 3). The differences between monoclinic and orthorhombic structures are smaller compared to the changes cause by chemical substitution.

The shift of the $t_{2 g}$ peak to the lower energies (decreasing gap between $e_{g}$ and $t_{2 g}$ states) is also mainly caused by the substitution of $\mathrm{Hf}$ with $\mathrm{Zr}$, while the sharpening of these features is a signature of additional structural transformations (especially sharpening of the first peak) as seen in our theoretical calculations (Figure 3).

Similar trends are found in the EXAFS spectra. The thin film spectra look comparable to the bulk reference spectra. While monoclinic $\mathrm{HfO}_{2}$ and the orthorhombic $\mathrm{Hf}_{0.5} \mathrm{Zr}_{0.5} \mathrm{O}_{2}$ films appear rather similar, there is an obvious difference to the tetragonal $\mathrm{Hf}_{0.25} \mathrm{Zr}_{0.75} \mathrm{O}_{2}$ and $\mathrm{ZrO}_{2}$ films.

For the $\mathrm{Hf}_{0.5} \mathrm{Zr}_{0.5} \mathrm{O}_{2}$ thin films both data ( $\mathrm{Zr}$ K-edge and $\mathrm{Hf} \mathrm{L}_{3}$-edge) correspond well to the model system with uniform distribution of $\mathrm{Zr}$ and $\mathrm{Hf}$ atoms in a monoclinic or orthorhombic lattice (Figure 2, Figure S13 and Figure S14). This can be well-explained by the difference between the gas phase deposition and the powder fabrication route: While in $\mathrm{ALD}, \mathrm{ZrO}_{2}$ and $\mathrm{HfO}_{2}$ sub-monolayers are deposited alternatingly, crystallites of $\mathrm{ZrO}_{2}$ and $\mathrm{HfO}_{2}$ are being mixed in the powder route. Before the high-temperature step (rapid thermal anneal or calcination), the distance that $\mathrm{Zr}$ as well as $\mathrm{Hf}$ atoms need to diffuse to establish a perfectly random distribution are about a factor of 100 larger (a few $\AA$ vs. a few tens of $\mathrm{nm}$ ). This suggests that powders are not always a very suitable reference system if the high-temperature step was not able to uniformly distribute $\mathrm{Hf}$ and $\mathrm{Zr}$ in the final product. We want to emphasize that this conclusion has impact that potentially extends beyond the system of hafnia-zirconia studies in this work.

For the $\mathrm{Hf}_{0.25} \mathrm{Zr}_{0.75} \mathrm{O}_{2}$ thin film, the $\mathrm{Hf}_{2}$-edge spectrum supports a tetragonal structure. We see in FT-EXAFS a double feature for the first shell of $\mathrm{Hf}(\mathrm{Hf}-\mathrm{O})$ which is characteristic for the tetragonal phase as follows from our theoretical calculations (Figure 1b). At the same time, the features of the second coordination shell are more disordered, which fits better to the model spectra (Figure 2) with significantly lower Zr concentration. In the tetragonal $\mathrm{Hf}_{0.25} \mathrm{Zr}_{0.75} \mathrm{O}_{2}$ model (Figure $\mathrm{S} 13$ ) compound, the $\mathrm{Hf}-\mathrm{Me}$ feature should be much more pronounced compared for other phases (e.g. monoclinic $\mathrm{HfO}_{2}$ and orthorhombic $\mathrm{Hf}_{0.5} \mathrm{Zr}_{0.5} \mathrm{O}_{2}$ ).

The $\mathrm{Zr}$ K-edge spectra of both $\mathrm{Hf}_{0.25} \mathrm{Zr}_{0.75} \mathrm{O}_{2}$ and $\mathrm{ZrO}_{2}$ thin films show features characteristic for the tetragonal phase. The difference in the amplitude of spectra is attributed to the chemical disorder mixing $\mathrm{Zr}$ and $\mathrm{Hf}$ as shown by our model calculations (Figure 2 and Figure S14). The only remarkable difference to the model calculations is that we do not see a double feature for $\mathrm{Zr}-\mathrm{O}$ in the first coordination shell, which might be a sign for dynamic correlation effects. The dynamic correlation effects might change the shape of radial distribution function (RDF) ${ }^{88}$. Another option is a pseudosymmetry with tetragonal lattice parameters and cubic oxygen positions as GIXRD mostly gives signal from metals.

Future studies will focus on how temperature dependent structure evolution is reflected in the spectra of thin film as well as powder reference samples. No temperature dependent data has been reported and analyzed even for the common bulk polymorphs such as the monoclinic, tetragonal or cubic phase. In thin films, an understanding of static and dynamic disorder is crucial as the situation with potentially multiple phases, size-effects, strain, vacancies or dopants is complex enough to pose a severe challenge. Advancing the understanding of the bulk phases will be the key to a full picture of what happens in these para-, ferro- and antiferroelectric thin films that are of great scientific and technological interest.

\section{SUPPORTING INFORMATION}

Details on the Rietveld refinement for the powder samples and the corresponding fit curves, a discussion of the XANES data for both bulk and thin films, the XANES and EXAFS results for the fluorescence mode measurement of the Si: $\mathrm{HfO}_{2}$ single-layer thin film sample and some additional EXAFS spectra of bulk and thin films can be found in the Supporting Information. This material is available free of charge via the Internet at http://pubs.acs.org.

\section{ACKNOWLEDGMENTS}


Björn Matthey (Fraunhofer IKTS, Dresden) is acknowledged for providing $\mathrm{HfO}_{2}$ and $\mathrm{ZrO}_{2}$ powders on short notice after DESY's renowned customs office punished us. Parts of this research were carried out at Petra III at DESY, a member of the Helmholtz Association (HGF). The experiments on single $\mathrm{Si}: \mathrm{HfO}_{2}$ thin film samples were performed at the CLAESS beamline at ALBA Synchrotron with the collaboration of ALBA staff. We would like to thank Edmund Welter for assistance (in using beamline P65) and DESY for enabling this research for proposal no. 20160591 and for travel support. T.S. acknowledges the German Research Foundation (DFG) for funding this work in the frame of the project "Inferox" (project no. MI 1247/11-2). B.J., J.L.J., and U.S. acknowledge funding from the Army Research Office through contract number W911NF-15-1-0593. This work was performed in part at the Analytical Instrumentation Facility (AIF) at North Carolina State University, which is supported by the State of North Carolina and the U.S. National Science Foundation (award number ECCS-1542015). The AIF is a member of the North Carolina Research Triangle Nanotechnology Network (RTNN), a site in the National Nanotechnology Coordinated Infrastructure $(\mathrm{NNCl})$.

\section{REFERENCES}

[1] H. H. Buckman, G. A. Pritchard, Highly-refractory article and method of producing the same - US Patent 1,375,077, 1921.

[2] J. B. Austin, The thermal expansion of some refractory oxides, 14, 11,795-810, 1931.

[3] V. V. Kharton, E. N. Naumovich, and A. A. Vecher. Research on the electrochemistry of oxygen ion conductors in the former Soviet Union. I. ZrO2 -based ceramic materials. Journal of Solid State Electrochemistry, 3(2):61-81, 1999.

[4] V. V. Kharton, A. A. Yaremchenko, E. N. Naumovich, and F. M. B. Marques. Research on the electrochemistry of oxygen ion conductors in the former Soviet Union III. HfO2-, CeO2- and ThO2-based oxides. Journal of Solid State Electrochemistry, 4(5):243-266, 2000.

[5] Garvie, R. C.; Hannink, R. H.; Pascoe, R. T., Ceramic steel?, Nature, 258(5537):703-704, 1975.

[6] Curtis, C. E., Development of Zirconia Resistant to Thermal Shock. Journal of the American Ceramic Society, 30(6):180-196, 1947.

[7] Curtis, C. E.; Doney, L. M.; Johnson, J. R., Some Properties of Hafnium Oxide, Hafnium Silicate, Calcium Hafnate, and Hafnium Carbide. J. Am. Cer. Soc., 37(10):458-465, 1954.

[8] Robertson, J., High dielectric constant oxides. Eur. Phys. J. Appl. Phys. 2004, 28(3), 265-291.

[9] Wilk, G. D., Wallace, R. M., \& Anthony, J. M., High-k gate dielectrics: Current status and materials properties considerations. J. Appl. Phys. 2001, 89(10), 5243-5275.

[10] Böscke, T. S.; Müller, J.; Bräuhaus, D.; Schröder, U.; Böttger, U. Ferroelectricity in Hafnium Oxide Thin Films. Appl. Phys. Lett. 2011, 99, 102903.

[11] Schroeder, U.; Yurchuk, E.; Müller, J.; Martin, D.; Schenk, T.; Polakowski, P.; Adelmann, C.; Popovici, M. I.; Kalinin, S. V.; Mikolajick, T. Impact of Different Dopants on the Switching Properties of Ferroelectric Hafniumoxide. Jpn. J. Appl. Phys. 2014, 53, 08LE02.

[12] Starschich, S., \& Boettger, U., An extensive study of the influence of dopants on the ferroelectric properties of HfO2. J. Mater. Chem. C 2017, 5(2), 333-338

[13] Park, M. H., Kim, H. J., Kim, Y. J., Jeon, W., Moon, T., Hwang, C. S., Ferroelectric properties and switching endurance of $\mathrm{Hf}_{0.5} \mathrm{Zr}_{0.5} \mathrm{O}_{2}$ films on TiN bottom and $\mathrm{TiN}$ or $\mathrm{RuO}_{2}$ top electrodes. Physica Status Solidi Rapid Research Letters 2014, 8(6), 532-535.

[14] Xu, L., Shibayama, S., Izukashi, K., Nishimura, T., Yajima, T., Migita, S., \& Toriumi, A., General relationship for cation and anion doping effects on ferroelectric HfO 2 formation. IEEE International Electron Devices Meeting (IEDM), 2016 pp. 25.2.1-25.2.4.

[15] Lomenzo, P. D.; Zhao, P.; Takmeel, Q.; Moghaddam, S.; Nishida, T.; Nelson, M.; Fancher, C. M.; Grimley, E. D.; Sang, X.; LeBeau, J. M.; Jones, J. L. Ferroelectric Phenomena in Si-Doped $\mathrm{HfO}_{2}$ Thin Films with TiN and Ir Electrodes. J. Vac. Sci. Technol., B: Nanotechnol. Microelectron.: Mater., Process., Meas., Phenom. 2014, 32, $03 D 123$.

[16] Shimizu, T.; Yokouchi, T.; Shiraishi, T.; Oikawa, T.; Sankara Rama Krishnan, P. S.; Funakubo, H. Study on the Effect of Heat Treatment Conditions on Metalorganic-Chemical-Vapor-deposited Ferroelectric Hf0.5Zr0.5O2 Thin Film on Ir Electrode, Jpn. J. Appl. Phys. 2014, 53, 09 PA04. 
[17] Florent, K., Lavizzari, S., Popovici, M., Di Piazza, L., Celano, U., Groeseneken, G., Van Houdt, J., Understanding ferroelectric Al: HfO2 thin films with Si-based electrodes for 3D applications. J. Appl. Phys. 2017, 121(20), 204103.

[18] Hoffmann, M.; Schroeder, U.; Schenk, T.; Shimizu, T.; Funakubo, H.; Sakata, O.; Pohl, D.; Drescher, M.; Adelmann, C.; Materlik, R.; Kersch, A.; Mikolajick, T. Stabilizing the Ferroelectric Phase in Doped Hafnium Oxide. J. Appl. Phys. 2015, 118 (7), 072006.

[19] Sharma, Y., Barrionuevo, D., Agarwal, R., Pavunny, S. P., \& Katiyar, R. S., Ferroelectricity in Rare-Earth Modified Hafnia Thin Films Deposited by Sequential Pulsed Laser Deposition. ECS Solid State Letters 2015, 4(11), N13N16.

[20] Lomenzo, P. D., Takmeel, Q., Fancher, C. M., Zhou, C., Rudawski, N. G., Moghaddam, S., Jones, J. L., Nishida, T. (2015). Ferroelectric Si-Doped HfO 2 Device Properties on Highly Doped Germanium. IEEE Electron Device Letters, 36(8), 766-768.

[21] J. Müller, E. Yurchuk, T. Schlösser, J. Paul, R. Hoffmann, S. Müller, D. Martin, S. Slesazeck, P. Polakowski, J. Sundqvist, M. Czernohorsky, K. Seidel, P. Kücher, R. Boschke, M. Trentzsch, K. Gebauer, U. Schröder, T. Mikolajick, Ferroelectricity in $\mathrm{HfO}_{2}$ enables nonvolatile data storage in $28 \mathrm{~nm}$ HKMG, IEEE Symposium on VLSI Technology (VLSIT), 2012, pp. 25-26, Honolulu, HI.

[22] J. Müller, T. S. Böscke, S. Muller, E. Yurchuk, P. Polakowski, J. Paul, D. Martin, T. Schenk, K. Khullar, A. Kersch, W. Weinreich, S. Riedel, K. Seidel, A. Kumar, T. M. Arruda, S. V. Kalinin, T. Schlösser, R. Boschke, R. van Bentum, U. Schröder, T. Mikolajick, Ferroelectric hafnium oxide: A CMOS-compatible and highly scalable approach to future ferroelectric memories. IEEE International Electron Devices Meeting (IEDM), 2013, Washington, DC, pp. 10.8.110.8.4.

[23] Starschich, S., Schenk, T., Schroeder, U., \& Boettger, U., Ferroelectric and piezoelectric properties of Hf1xZrxO2 and pure ZrO2 films. Appl. Phys. Lett. 2017, 110(18), 182905.

[24] Smith, S. W., Kitahara, A. R., Rodriguez, M. A., Henry, M. D., Brumbach, M. T., \& Ihlefeld, J. F. (2017). Pyroelectric response in crystalline hafnium zirconium oxide (Hf1-x Zr x O2) thin films. Applied Physics Letters, 110(7), 072901.

[25] Jachalke, S.; Schenk, T.; Mehner, E. Schröder, U.; Stöcker, H.; Mikolajick, T.; Meyer, D. C.; Pyroelectricity of silicon-doped hafnium oxide, Appl. Phys. Lett. 2018, 112(14), 142901.

[26] Sang, X.; Grimley, E. D.; Schenk, T.; Schroeder, U.; LeBeau, J. M. On the Structural Origins of Ferroelectricity in HfO2 Thin Films. Appl. Phys. Lett. 2015, 106, 162905.

[27] Shimizu, T., Katayama, K., Kiguchi, T., Akama, A., Konno, T. J., \& Funakubo, H., Growth of epitaxial orthorhombic YO1.5-substituted $\mathrm{HfO}_{2}$ thin film. Applied Physics Letters 2015, 107(3), 032910.

[28] Polakowski, P.; Riedel, S.; Weinreich, W.; Rudolf, M.; Sundqvist, J.; Seidel, K.; Muller, J. Ferroelectric Deep Trench Capacitors Based on Al:HfO2 for 3D Nonvolatile Memory Applications. In 2014 IEEE 6th International Memory Workshop (IMW); Taipei, Taiwan, May 18-21, 2014 ; IEEE: Piscataway, NJ, 201410.1109/IMW.2014.6849367.

[29] Park, M. H., Kim, H. J., Kim, Y. J., Moon, T., Do Kim, K., Hwang, C. S., Toward a multifunctional monolithic device based on pyroelectricity and the electrocaloric effect of thin antiferroelectric HfxZr1-xO2 films. Nano Energy 2015, 12, 131-140.

[30] Park, M. H., Kim, H. J., Kim, Y. J., Moon, T., Kim, K. D., \& Hwang, C. S. , Thin HfxZr1-xO2 Films: A New LeadFree System for Electrostatic Supercapacitors with Large Energy Storage Density and Robust Thermal Stability. Adv. Energy Mater. 2014, 4(16).

[31] Ali, F., Liu, X., Zhou, D., Yang, X., Xu, J., Schenk, T., Müller, J., Schroeder, U., Cao, F., Dong, X. Silicon-doped hafnium oxide anti-ferroelectric thin films for energy storage. J. Appl. Phys. 2017, 122(14), 144105.

[32] Pešić, M., Hoffmann, M., Richter, C., Mikolajick, T., \& Schroeder, U., Nonvolatile Random Access Memory and Energy Storage Based on Antiferroelectric Like Hysteresis in $\mathrm{ZrO}_{2}$. Adv. Funct. Mater. 2016, 26(41), 7486-7494.

[33] Mulaosmanovic, H., Ocker, J., Müller, S., Noack, M., Müller, J., Polakowski, P., Mikolajick, T., Slesazeck, S., Novel ferroelectric FET based synapse for neuromorphic systems. IEEE Symposium on VLSI Technology, 2017 (pp. T176-T177). 


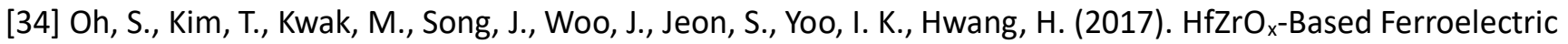
Synapse Device With 32 Levels of Conductance States for Neuromorphic Applications. IEEE Electron Device Letters, 38(6), 732-735.

[35] Hoffmann, M., Pešić, M., Chatterjee, K., Khan, A. I., Salahuddin, S., Slesazeck, S., Schroeder, U., Mikolajick, T., Direct observation of negative capacitance in polycrystalline ferroelectric $\mathrm{HfO}_{2}$. Adv. Funct. Mater. 2016, 26(47), 8643-8649.

[36] Ambriz-Vargas, F., Kolhatkar, G., Broyer, M., Hadj-Youssef, A., Nouar, R., Sarkissian, A., Thomas, R., Gomez-

[37] Dragoman, M.; Modreanu, M.; Povey, I. M.; lordanescu, S.; Aldrigo, M.; Romanitan, C.; Vasilache, D.; Dinescu, A.; Dragoman, D., Very large phase shift of microwave signals in a $6 \mathrm{~nm} \mathrm{Hf}_{x} \mathrm{Zr}_{1-\mathrm{x}} \mathrm{O}_{2}$ ferroelectric at $\pm 3 \mathrm{~V}$. Nanotechnology 2017, 28(38), 38LT04.

[38] Dragoman, M., Aldrigo, M., Modreanu, M., Dragoman, D., Extraordinary tunability of high-frequency devices using Hf0.3Zr0.7O2 ferroelectric at very low applied voltages. Applied Physics Letters 2017, 110(10), 103104.

Yanez, C., Gauthier, M. A., Ruediger, A., A Complementary Metal Oxide Semiconductor Process-Compatible Ferroelectric Tunnel Junction. ACS Appl. Mater. Inter. 2017, 9(15), 13262-13268.

[39] Materlik, R.; Künneth, C.; Kersch, A. The Origin of Ferroelectricity in Hf0.5ZrO.5O2: A Computational Investigation and a Surface Energy Model. J. Appl. Phys. 2015, 117, 134109.

[40] Batra, R., Tran, H. D., Ramprasad, R., Stabilization of metastable phases in hafnia owing to surface energy effects. Appl. Phys. Lett. 2016, 108(17), 172902.

[41] Park, M. H., Lee, Y. H., Kim, H. J., Schenk, T., Lee, W., Do Kim, K., Fengler, F. P. G., Mikolajick, T., Schroeder, U., Hwang, C. S., Surface and grain boundary energy as the key enabler to ferroelectricity in nanoscale hafniazirconia: comparison of model and experiment. Nanoscale2017, 9, 9973-9986.

[42] T. Shiraishi, K. Katayama, T. Yokouchi, T. Shimizu, T. Oikawa, O. Sakata, H. Uchida, Y. Imai, T. Kiguchi, T. J. Konno, H. Funakubo, Effect of the film thickness on the crystal structure and ferroelectric properties of (Hf0.5Zr0.5)O2 thin films deposited on various substrates, Mater. Sci. in Semicon. Proc. 70, 239-245, 2017.

[43] Y. Wei, P. Nukala, M. Salverda, S. Matzen, H. J. Zhao, J. Momand, A. Everhardt, G. R. Blake, P. Lecoeur, B. J. Kooi, J. Íñiguez, B. Dkhil, B. Noheda, A rhombohedral ferroelectric phase in epitaxially-strained $\mathrm{Hf}_{0.5} \mathrm{Zr}_{0.5} \mathrm{O}_{2}$ thin films, Nature Mater., 17, 1095-1100, 2018.

[44] U. Schroeder, C. Richter, M. H. Park, T. Schenk, M. Pešić, M. Hoffmann, F. P. G. Fengler, D. Pohl, B. Rellinghaus, C. Zhou, C.-C. Chung, J. L. Jones, T. Mikolajick, Lanthanum-Doped Hafnium Oxide: A Robust Ferroelectric Material, Inorg. Chem., 57, 5, 2752-2765, 2018.

[45] C. Richter, T. Schenk, M. H. Park, F. A. Tscharntke, E. D. Grimley, J. M. LeBeau, C. Zhou, C. M. Fancher, J. L. Jones, T. Mikolajick, U. Schroeder, Si doped hafnium oxide-A "fragile" ferroelectric system, Adv. Electronic Mater. 3, 10, 1700131, 2017.

[46] Reyes-Lillo, S. E., Garrity, K. F., \& Rabe, K. M. Antiferroelectricity in thin-film ZrO2 from first principles. Phys. Rev. B 2014, 90(14), 140103.

[47] Huan, T. D., Sharma, V., Rossetti Jr, G. A., \& Ramprasad, R. Pathways towards ferroelectricity in hafnia. Phys. Rev. B 2014, 90(6), 064111.

[48] Batra, R., Huan, T. D., Jones, J. L., Rossetti Jr, G., \& Ramprasad, R. (). Factors Favoring Ferroelectricity in Hafnia: A First-Principles Computational Study. J. Phys. Chem.C 2017, 121(8), 4139-4145.

[49] Grimley, E. D., Schenk, T., Sang, X., Pešić, M., Schroeder, U., Mikolajick, T., \& LeBeau, J. M., Structural Changes Underlying Field-Cycling Phenomena in Ferroelectric $\mathrm{HfO}_{2}$ Thin Films. Adv. Electron. Mater. 2016, 2(9).

[50] Grimley, E. D., Schenk, T., Mikolajick, T., Schroeder, U., LeBeau, J. M. Atomic Structure of Domain and Interphase Boundaries in Ferroelectric $\mathrm{HfO}_{2}$. Adv. Mater. Interfaces 2018, 5 (5), 1701258.

[51] J. J. Rehr and R. C. Albers, Theoretical Approaches to X-ray Absorption Fine Structure. Rev. Mod. Phys. 2000, $72,621$.

[52] Timoshenko, J.; Anspoks, A.; Kalinko, A.; Kuzmin, A.; Thermal disorder and correlation effects in anti-perovskite-type copper nitride, Acta Mater. 2017, 129, 61-71.

[53] Kuzmin, A.; Anspoks, A.; Kalinko, A.; Timoshenko, J.; The use of x-ray absorption spectra for validation of classical force-field models, Z. Phys. Chem. 2016, 230, 537-549. 
[54] V. L. Aksenov, M. V. Koval'chuk, A. Yu. Kuz'min, Yu. Purans, S. I. Tyutyunnikov, Development of methods of EXAFS spectroscopy on synchrotron radiation beams. Crystallogr. Rep. 2006, 51, 908-935

[55] Schenk, T., Hoffmann, M., Ocker, J., Pešić, M., Mikolajick, T., Schroeder, U. (2015). Complex internal bias fields in ferroelectric hafnium oxide. ACS Appl. Mater. Interfaces, 7(36), 20224-20233.

[56] Pešić, M., Fengler, F. P. G., Larcher, L., Padovani, A., Schenk, T., Grimley, E. D., Sang, X., LeBeau, J. M., Slesazeck, S., Schroeder, U. Mikolajick, T., Physical Mechanisms behind the Field-Cycling Behavior of $\mathrm{HfO}_{2}-\mathrm{Based}^{-}$ Ferroelectric Capacitors. Adv. Funct. Mater. 2016, 26(25), 4601-4612.

[57] Ping Li, I-Wei Chen and J-E. Penner-Hahn, X-ray-absorption studies of zirconia polymorphs. I. Characteristic local structures, PRB, 48, pp:10063, 1993; DOI 10.1103/PhysRevB.48.10063;

[58] Krug C.; Lucovsky G.; Spectroscopic characterization of high k dielectrics: Applications to interface electronic structure and stability against chemical phase separation. Journal of Vacuum Science \& Technology A 2004, 22, 1301-1308. DOI: 10.1116/1.1755714

[599 J. Morais, L. Miotti, K. P. Bastos, S. R. Teixeira, I. J. R. Baumvol, A. L. P. Rotondaro, J. J. Chambers, M. R. Visokay, L. Colombo, and M. C. Martins Alves; Environment of hafnium and silicon in Hf-based dielectric films: An atomistic study by $\mathrm{x}$-ray absorption spectroscopy and x-ray diffraction. Appl. Phys. Lett. 2005, 86, 212906; doi: $10.1063 / 1.1935042$

[60] N.D. Afifya; G. Dalbaa; U. Mahendra Kumar Koppolu; C. Armellini; Y. Jestin; F. Rocca; XRD and EXAFS studies of $\mathrm{HfO} 2$ crystallisation in SiO2-HfO2 films. Materials Science in Semiconductor Processing 9 (2006) 1043-1048. doi:10.1016/j.mssp.2006.10.021

[61] Cho D.-Y.; Jung H.-S.; Hwang C. S.; Structural properties and electronic structure of HfO2-ZrO2 composite films. Phys. Rev. B 2010, 82, 094104. DOI: 10.1103/PhysRevB.82.094104

[62] Sahiner M. A.; Lysaght P. S.; Price J.; Kirsch P. D.; Woicik J. C.; Klump A.; Reehil C.; Manners W. A.; Zr induced structural changes in Hf1-xZrxO2 high-k thin films. Appl. Phys. A (2014) 117, 93-96; DOI: 10.1007/s00339-0138208-z

[63] Sharma A; Varshney M.; Kang S.; Baik J.; Ha T.-K.; Chae K.-H.; Kumar S.; Shin H.-J.; Electronic structure study and dielectric properties of amorphous $\mathrm{ZrO} 2$ and HfO2. Adv. Mater. Lett. 2016, 7(1), 17-22.

[64] Ping Li, I-Wei Chen and J-E. Penner-Hahn, X-ray-absorption studies of zirconia polymorphs. III. Static distortion and thermal distortion, PRB, 48, pp:10082, 1993; DOI 10.1103/PhysRevB.48.10082

[65] Ping Li, I-Wei Chen and J-E. Penner-Hahn, X-ray-absorption studies of zirconia polymorphs. II. Effect of Y2O3 dopant on ZrO2 structure, PRB, 48, pp:10074, 1993; DOI 10.1103/PhysRevB.48.10063

[66] Ping Li, I-Wei Chen and J-E. Penner-Hahn, Ef-fect of Dopants on Zirconia Stabilization-An X-ray Absorption Study: I, Trivalent dopants, J. Am. Chem. Soc., 77, 118 (1994); DOI 10.1111/j.1151-2916.1994.tb06964.x

[67] Ping Li, I-Wei Chen and J-E. Penner-Hahn, Effect of Dopants on Zirconia Stabilization-An X-ray Absorption Study: II, Tetravalent dopants, J. Am. Chem. Soc., 77, 1281 (1994); DOI 10.1111/j.1151-2916.1994.tb05403.x

[68] Ping Li, I-Wei Chen and J-E. Penner-Hahn, Effect of Dopants on Zirconia Stabilization-An X-ray Absorption Study: III, Charge compensating dopants, J. Am. Chem. Soc., 77, 1289 (1994); DOI 10.1111/j.11512916.1994.tb05404.x

[69] Booth, C. H.; Bridges, F.; Improved self-absorption correction for fluorescence measurements of extended x-ray absorption fine-structure. Physica Scripta, 2005(T115):202, 2005. doi:10.1238/Physica.Topical.115a00202.

[70] Rehr, J.; Kas, J.J.; Vila, F.D.; Prange, M.P.; Jorissen, K.; Parameter-free calculations of $x$-ray spectra with FEFF9. Phys. Chem. Chem. Phys., 2010, 12, 5503-5513.

[71] Schenk, T. Formation of ferroelectricity in hafnium based thin films, Ph. D. Thesis, Technische Universität Dresden, Germany (2016).

[72] Schenk, T.; Fancher, C. M.; Park, M. H.; Richter, C.; Künneth, C.; Kersch, A.; Jones, J. L. ; Mikolajick, T.; Schroeder, U., On the Origin of the Large Remanent Polarization in La: $\mathrm{HfO}_{2}$, Adv. Electron. Mater. (accepted for publication).

[73] Schenk, T.; Fancher, C. M.; Park, M. H.; Starschich, S.; Böttger, U.; Mikolajick, T.; Schroeder, U., Relaxation of Stress with Thickness in Ferroelectric ZrO2 Films (in review).

[74] S. Choi; T. Shiraishi; T. Kiguchi; T. Shimizu; Funakubo, H.; Konno, T. J., Formation of polar phase in Fe-doped ZrO2 epitaxial thin films, Appl. Phys. Lett. (2018), 113, 262903. 
[75] O. Bunau, Y. Joly, Self-consistent aspects of x-ray absorption calculations, J. Phys. : Condens. Matter 21, 345501 (2009).

[76] T. Mittmann, F. P. G. Fengler, C. Richter, M. H. Park, T. Mikolajick, U. Schroeder, Optimizing process conditions for improved Hf1 - xZrxO2 ferroelectric capacitor performance, Microelectron. Eng. 2017, 178, 48-51.

[77] Senf, F.; Flechsig, U.; Eggenstein, F.; Gudat, W.; Klein, R.; Rabus, H.; Ulm, G.; A plane-grating monochromator beamline for the PTB undulators at BESSY II, J. Synchrotron Rad. (1998) 5, 780-782

[78] Scholze, F.; Beckhoff, B. ;Brandt, G.;Fliegauf, F.; Gottwald, A.;Klein, R.; Meyer, B.; Rost, D.; Schwarz, U.; Thornagel, R.; Tümmler, J.; Vogel, K.; Weser, J.; Ulm, G.; High-accuracy EUV metrology of PTB using synchrotron radiation, Proc. SPIE, vol. 4344, pp. 402-413, 2001.]

[79] Lubeck, J.; Beckhoff, B.; Fliegauf, R.; Holfelder, I.; Hönicke, P.; Müller, M.; Pollakowski, B.; Reinhardt, F.; Weser, J.; A novel instrument for quantitative nanoanalytics involving complementary X-ray methodologies, Rev. Sci. Instrum. 84, 045106 (2013).

[80] L. Simonelli, C. Marini, W. Olszewski, M. Avila Perez, N. Ramanan, G. Guilera, V. Cuartero, and K. Klementiev, CLÆESS: the hard x-ray absorption beamline of the ALBA CELLS synchrotron, Cogent Phys., 3, 1231987, 2016.

[81] B. Ravel and M. Newville, ATHENA, ARTEMIS, HEPHAESTUS: data analysis for X-ray absorption spectroscopy using IFEFFIT. Journal of Synchrotron Radiation 2005, 12, 537-541. doi:10.1107/S0909049505012719

[82] Doebelin, N., Kleeberg, R., Profex: a graphical user interface for the Rietveld refinement program BGMN, J. Appl. Cryst. 2015. 48, 1573-1580.

[83] Brian H. Toby, R factors in Rietveld analysis: How good is good enough?, Powder Diffraction, 21, 1, 67-70, 2006.

[84] Ruh, R., Garrett, H. J., Domagala, R. F., Tallan, N.. The Svstern Zirconia-Hafnia. Journal of the American Ceramic Society (1968), 51(1), 23-28.

[85] Müller, J.; Böscke, T. S.; Schröder, U.; Mueller, S.; Bräuhaus, D.; Böttger, U.; Frey, L.; T. Mikolajick, Ferroelectricity in Simple Binary $\mathrm{ZrO}_{2}$ and $\mathrm{HfO}_{2}$, Nano Lett., 2012, 12 (8), 4318-4323.

[86] Erenburg, S. B.; Trubina, S. V. ; Kvashnina, K. O.; Kruchinin, V. N. ; Gritsenko, V. V. ; Chernikova, A. G. ; Markeev, A. M., Short-Range Order in Amorphous and Crystalline Ferroelectric Hf0.5Zr0.5O2, Journal of Experimental and Theoretical Physics (2018), 126(6), 816-824.

[87] B. Pollakowski, B. Beckhoff, F. Reinhardt, S. Braun, P. Gawlitza, Speciation of deeply buried TiOx nanolayers with grazing-incidence $x$-ray fluorescence combined with a near-edge $x$-ray absorption fine-structure investigation, Phys. Rev. B, 77, 235408, 2008.

[88] A. Anspoks, C. Marini, T. Miyanaga, B. Joseph, A. Kuzmin, J. Purans, J. Timoshenko, A. Bussmann-Holder, Local structure of $A$-atom in $\mathrm{ABO}_{3}$ perovskites studies by RMC-EXAFS, Rad. Phys. Chem. (2019) 10.1016/j.radphyschem.2018.11.026

[89] Barabash, S. V., Pramanik, D., Zhai, Y., Magyari-Kope, B., Nishi, Y. Ferroelectric switching pathways and energetics in (Hf,Zr)O $\mathrm{O}_{2}$. ECS Trans. 2017, 75(32), 107-121.

[90] Clima, S., Wouters, D. J., Adelmann, C., Schenk, T., Schroeder, U., Jurczak, M., Pourtois, G. Identification of the ferroelectric switching process and dopant-dependent switching properties in orthorhombic HfO2: A first principles insight. App. Phys. Lett. 2014, 104(9), 092906. 